\title{
Suppression of camptothecin biosynthetic genes results in metabolic modification of secondary products in hairy roots of Ophiorrhiza
} pumila

Takashi Asano $^{\text {a,b, },}$, Kanae Kobayashi ${ }^{a}$, Emi Kashihara ${ }^{a}$, Hiroshi Sudo ${ }^{\text {a,2 }}$, Ryosuke Sasaki ${ }^{\mathrm{c}}$, Yoko Iijima $^{\mathrm{c}, 3}$, Koh Aoki ${ }^{\mathrm{c}, 4}$, Daisuke Shibata ${ }^{\mathrm{c}}$, Kazuki Saito, ${ }^{\mathrm{a}, \mathrm{d},{ }^{*}}$, and Mami Yamazaki ${ }^{\mathrm{a}, \mathrm{b},{ }^{*}}$

${ }^{\mathrm{a}}$ Graduate School of Pharmaceutical Sciences, Chiba University, Inohana 1-8-1, Chuo-ku, Chiba 260-8675, Japan

${ }^{\mathrm{b}}$ CREST, Japan Science and Technology Agency, 4-1-8 Honcho, Kawaguchi, Saitama 332-0012, Japan

${ }^{\mathrm{c}}$ Kazusa DNA Research Institute, 2-6-7 Kazusa-Kamatari, Kisarazu, Chiba 292-0818, Japan

${ }^{\mathrm{d}}$ RIKEN Plant Science Center, 1-7-22 Suehiro-cho, Tsurumi-ku, Yokohama 230-0045, Japan

${ }^{*}$ Corresponding authors: K. Saito, M. Yamazaki

E-mail: ksaito@faculty.chiba-u.jp,mamiy@faculty.chiba-u.jp

Address: Graduate School of Pharmaceutical Sciences, Chiba University, Inohana 1-8-1, Chuo-ku, Chiba 260-8675, Japan

Tel.: +81-43-226-2931

(C) 2012. This manuscript version is made available under the Elsevier user license http://www.elsevier.com/open-access/userlicense/1.0/ 
${ }^{1}$ Present address: School of Pharmacy, Iwate Medical University, 2-1-1 Nishitokuta, Yahaba, Iwate 028-3694, Japan

${ }^{2}$ Present address: Faculty of Pharmaceutical Sciences, Hoshi University, 2-4-41 Ebara, Shinagawa-ku, Tokyo 142-8501, Japan

${ }^{3}$ Present address: Department of Nutrition and Life Science, Kanagawa Institute of Technology, 1030 Shimo-ogino, Atsugi, Kanawaga 243-0292, Japan

${ }^{4}$ Present address: Graduate School of Life and Environmental Sciences, Osaka Prefecture University, 1-1 Gakuen-cho, Naka-ku, Sakai, Osaka 599-8531, Japan 


\begin{abstract}
Camptothecin is a monoterpenoid indole alkaloid that exhibits anti-tumor activity. In Ophiorrhiza pumila, production of camptothecin and its related alkaloids was high in the hairy roots, but not in the cell suspension culture derived from hairy roots. To identify the intermediates in camptothecin biosynthesis, expression of genes encoding tryptophan decarboxylase (TDC) and secologanin synthase (SLS), the 2 enzymes catalyzing the early steps in camptothecin biosynthesis, were suppressed in the hairy roots of O. pumila by RNA interference (RNAi), and metabolite changes were investigated. In most $T D C$ - and $S L S$-suppressed lines, accumulation of camptothecin and related alkaloids, strictosidine, strictosamide, pumiloside, and deoxypumiloside was reduced. The accumulation levels of secologanin exhibited a strong negative correlation with the expression level of $T D C$, and that of loganin exhibited a negative correlation with the expression level of $S L S$. Some hairy root-specific chromatographic peaks detected by liquid chromatography Fourier transform ion cyclotron resonance mass spectrometry (LC-FTICR-MS) exhibited positive or negative correlation with TDC expression, suggesting their possible involvement in camptothecin biosynthesis.
\end{abstract}




\section{Keywords}

Ophiorrhiza pumila; Rubiaceae; Hairy root; RNAi; FTICR-MS; LC-FTICR-MS;

Monoterpenoid indole alkaloids; Camptothecin; Tryptophan decarboxylase (TDC);

Secologanin synthase (SLS) 


\section{Introduction}

A success story of alkaloids began with the discovery of nitrogen-containing plant products 200 years ago (Zenk and Juenger, 2007). Alkaloids are basic nitrogen-containing compounds found in about $20 \%$ of all plant species (Facchini and De Luca, 2008). Many alkaloids are pharmacologically active and have traditionally been used as treatments for various diseases (Kutchan, 1995). Camptothecin is a well-known alkaloid biosynthetically belonging to the monoterpenoid indole family, and was originally identified in the extracts of a Chinese tree Camptotheca acuminata (Wall et al., 1966). It exhibits anti-tumor activity by inhibiting topoisomerase-I, thereby killing cancer cells (Hsiang et al., 1985). At present, semi-synthetic water-soluble camptothecin derivatives such as topotecan and irinotecan are used as clinical anti-tumor agents against cancers of the lung, cervix, ovaries, colon, and other organs throughout the world (Pizzolato and Saltz, 2003). Consequently, the demand for camptothecin will increase in the future. In spite of the rapid growth of the pharmaceutical market, camptothecin is exclusively produced from intact plants, mainly C. acuminata and Nothapodytes foetida (Govindachari and Viswanathan, 1972).

Because of the shortage of natural resources and the associated environmental concerns, production of secondary metabolites by genetically engineered plant cell cultures has 
become a keen issue (Oksman-Caldentey and Saito, 2005; Sirikantaramas et al., 2007).

Camptothecin and related alkaloids are produced in relatively wide array of plant species belonging to different families, such as Nyssaceae, Icacinaceae, Rubiaceae, and Apocynaceae. In spite of many trials, it is still difficult to obtain tissue cultures or transgenic plants in these species. We have performed genetic transformation of Ophiorrhiza species, including O. pumila, O. liukiuensis, and O. kuroiwai, and succeeded in establishing hairy root cultures (Asano et al., 2004; Saito et al., 2001; Watase et al., 2004), which produce a good yield of camptothecin and the related alkaloids, namely, strictosamide, pumiloside, and deoxypumiloside (Asano et al., 2004; Kitajima et al., 2002; Yamazaki et al., 2003a).

Monoterpenoid indole alkaloids (TIAs), including camptothecin, are derived from strictosidine, which is a common intermediate formed by the condensation of tryptamine, an indole compound, with secologanin, an iridoid glucoside, catalyzed by strictosidine synthase (STR) (Kutchan et al., 1988; Stöckigt et al., 1977; Yamazaki et al., 2004) (Fig. 1). Tryptamine is formed by decarboxylation of tryptophan catalyzed by tryptophan decarboxylase (TDC) (De Luca et al., 1989; Noé et al., 1984). The other precursor, secologanin, is biosynthesized from loganin by secologanin synthase (SLS) (Yamamoto et al., 2000). The gene CYP72A1 from Catharanthus roseus was identified 
to encode SLS (Irmler et al., 2000). Using this sequence information, the cDNAs encoding STR and TDC were cloned from hairy roots of O. pumila (Yamazaki et al., 2003b).

Intramolecular cyclization of strictosidine produces strictosamide, an intermediate in camptothecin biosynthesis, as proven by incorporation of radiolabeled precursor (Hutchinson et al., 1979). Possible precursors of camptothecin, such as pumiloside and 3(S)-deoxypumiloside, were isolated from Ophiorrhiza species (Aimi et al., 1989; Kitajima et al., 2005), and pumiloside has also been found in C. acuminata (Carte et al., 1990). However, intermediates in the later steps after strictosamide generation in camptothecin biosynthesis have not been identified.

We have used RNA interference (RNAi) technology as an effective way to investigate camptothecin biosynthesis. In this study, hairy roots of $O$. pumila were suppressed for the expression of $T D C$ and $S L S$ using RNAi, and the accumulation patterns of camptothecin and related alkaloids were correlated with the expression levels of the targeted genes in RNAi hairy roots. Cell suspension cultures induced from hairy roots did not accumulate alkaloids. Specific metabolites in $O$. pumila hairy roots were selected by comparing the metabolite patterns of hairy roots and cell suspension cultures using Fourier transform ion cyclotron resonance mass spectrometry 
1

2

3 4 5

(FTICR-MS). From these specific metabolites, candidates for intermediates in camptothecin biosynthesis were selected based on their metabolic changes in RNAi hairy roots. 


\section{Results and Discussion}

\subsection{Establishment of cell suspension culture induced from hairy roots}

O. pumila hairy roots produce a large amount of camptothecin and a variety of

related alkaloids (Saito et al., 2001; Yamazaki et al., 2003a). Light yellow calli induced from leaves and shoots of $O$. pumila plants do not produce alkaloids, but accumulate a variety of anthraquinones (Kitajima et al., 1998). In this study, new callus was induced from O. pumila hairy roots to produce camptothecin, and was subcultured in liquid medium in the dark to establish a cell suspension culture. The accumulation patterns of secondary metabolites in cell suspension culture were profiled by HPLC-photodiode array detection-electrospray ionization mass spectrometer (HPLC/DAD/ESI/MS) and compared with those of hairy roots. Hairy roots produced many alkaloids and anthraquinones; however, the cell suspension culture accumulated no alkaloids and little anthraquinones (Fig. 2). The accumulation of camptothecin in cell suspension culture was also not confirmed by fluorescence-HPLC analysis (data not shown).

\subsection{Establishment of RNAi hairy roots}

To investigate the modulation of camptothecin biosynthesis using RNAi 
technology, $T D C$ and $S L S$ were chosen as target genes, because these 2 genes are known to be involved in camptothecin biosynthesis. For induction of RNAi hairy roots, the stem segments of young aseptic plants of O. pumila were infected with Agrobacterium rhizogenes 15834 harboring the RNAi plasmid pGWB80-TDCi or pGWB80-SLSi. Fifteen TDCi hairy root (TDCi-HR) lines and 16 SLSi hairy root (SLSi-HR) lines were established. As a negative control, hairy roots harboring pGWB80-GUSi were established (GUSi-HR). To evaluate the suppression of $T D C$ and $S L S$ genes in TDCi-HR and SLSi-HR, respectively, gene expression was analyzed in RNAi hairy roots by semi-quantitative RT-PCR. TDC expression was downregulated in TDCi-HR, and 5 TDCi-HR lines expressed less than $40 \%$ compared to the average expression of $T D C$ in the GUSi-HR control (Fig. 3A). Similarly, SLS expression was downregulated in SLS-HR, and $7 \mathrm{SLSi-HR}$ lines expressed less than $80 \%$ compared to the average expression in GUSi-HR (Fig. 3B).

\subsection{Changes in accumulation patterns of camptothecin-related alkaloids in RNAi hairy roots}

The camptothecin content in RNAi hairy roots used for gene expression analysis was quantified by fluorescence-HPLC. As seen in Fig. 3, camptothecin 
production was suppressed in TDCi-HR and SLSi-HR, and the suppression positively correlated with the targeted gene expression levels. In most $T D C$ - and $S L S$-suppressed lines, the production of camptothecin was about one-half of that of the average in GUSi-HR. Furthermore, metabolite profiling of TDCi-HR and SLSi-HR by HPLC/DAD/ESI/MS indicated that the production of strictosamide, pumiloside, and other unknown compounds with similar eluting times was suppressed (Fig. 4). These unknown compounds may be the candidates for intermediates in camptothecin biosynthesis.

\subsection{Non-targeted metabolite analysis by infusion FTICR-MS}

We identified potential candidates for post-strictosamide intermediates in camptothecin biosynthesis by non-targeted metabolite analysis of hairy roots and camptothecin non-producing cell suspension culture using infusion FTICR-MS. In this analysis, 832 and 659 ion peaks were detected in hairy roots and cell suspension culture, respectively. The $m / z$ values for the detected ion peaks ranged from 116 to 1800 . To identify the principal candidates, differentially expressed peaks between hairy root and cell suspension culture were narrowed down. By this process, 241 of the 832 peaks in hairy roots were categorized as hairy root-specific peaks (Fig. 5), which included peaks 
corresponding to camptothecin, strictosidine and the camptothecin-related alkaloids such as strictosamide, pumiloside, and deoxypumiloside.

\subsection{Semi-quantitative mass analysis of camptothecin-related alkaloids in RNAi}

\section{hairy roots}

As previously mentioned, the production of camptothecin and its related alkaloids strictosamide and pumiloside were suppressed in TDCi-HR and SLSi-HR. Camptothecin content was quantified by fluorescence-HPLC; however, other camptothecin-related alkaloids were impossible to quantify using the same method, because these alkaloids lacked specific fluorescence and were co-eluted with several compounds in an HPLC separation detected by photodiode array. As a comprehensive method of targeted metabolite quantification, the peak area of a mass signal acquired by LC-FTICR-MS analysis was used. As the FTICR-MS analysis provides mass values with very high accuracy and resolution, the greater part of metabolites are acquired as a single mass peak. We used this method for the identification and semi-quantification of the targeted metabolites. As seen in Fig. 6, the production of camptothecin, strictosidine, and the camptothecin-related alkaloids, such as strictosamide, pumiloside, and deoxypumiloside, was suppressed, and the suppression was positively correlated with 
the expression levels of $T D C$ and $S L S$. Strictosidine, strictosamide, and pumiloside, in particular, exhibited a strong correlation.

\subsection{Semi-quantitative analysis of secologanin biosynthesis intermediates in RNAi hairy roots}

To understand the effect of targeted gene suppression on pre-strictosidine events, we examined the changes in accumulation of secologanin and its biosynthetic intermediates in RNAi hairy roots. Secologanin accumulated in TDCi-HR, and exhibited a strong negative correlation with $T D C$ expression. Loganin accumulated in SLSi-HR and exhibited a negative correlation with $S L S$ expression (Fig. 7). Furthermore, we examined the accumulation changes in RNAi hairy roots for peaks of accurate mass corresponding to the predicted intermediates in secologanin biosynthesis (Fig. 8). Other intermediates, such as 7-deoxyloganic acid and loganic acid, did not exhibit a correlation with $T D C$ and $S L S$ expression (Fig. 7).

\subsection{Narrowing down of new candidates for intermediates in camptothecin biosynthesis}

The 241 hairy root-specific peaks obtained by non-targeted metabolite analysis 
using infusion FTICR-MS should include many possible intermediates in camptothecin biosynthesis. Because the production of camptothecin-related alkaloids, such as strictosidine, strictosamide and pumiloside, were significantly suppressed in TDCi-HR, hairy root-specific peaks that exhibit a good correlation with $T D C$ expression represent potential candidates for intermediates in camptothecin biosynthesis. To effectively narrow down the candidates, we selected the potential peaks related to camptothecin biosynthesis from the 241 hairy root-specific peaks according to the schematic flow shown in Fig. 5. Twelve chromatographic peaks exhibited positive correlation, and 11 chromatographic peaks exhibited negative correlation with $T D C$ expression.

Among the hairy root-specific peaks that exhibited positive or negative correlations with $T D C$ expression, probable metabolites were annotated based on elemental composition calculated from accurate $\mathrm{m} / \mathrm{z}$ values using the public database KNApSAcK (http://kanaya.naist.jp/KNApSAcK) (Afendi et al., in press). Among the peaks with positive correlation, 2 peaks had probable annotations of secondary metabolites (Table 1). Gelsemicine is an indole alkaloid isolated from Gelsemium species (Kitajima et al., 2006; Przybylska and Marion, 1961). Lyaloside is an indole alkaloid isolated from Pauridiantha lyalli (Aimi et al., 1992). Although a direct comparison with standard compounds revealed that these 2 peaks were not due to these 
annotated compounds, these compounds may be potential biosynthetic intermediates with identical chemical composition. Among the peaks with negative correlation, 6 peaks were annotated as flavonoids (Table 2). Many peaks were not annotated by the KNApSAcK search; however, two non-annotated peaks had exact masses almost the same as those of the possible intermediates in camptothecin biosynthesis (Fig. 9). 


\section{Conclusions}

The production of camptothecin, strictosidine, and camptothecin-related

alkaloids, such as strictosamide, pumiloside, and deoxypumiloside, was suppressed in a $T D C$ and $S L S$ expression-dependent manner in RNAi hairy roots. Among the hairy root-specific peaks that correlated with $T D C$ expression in LC-FTICR-MS analysis, 2 unknown peaks with positive correlation were annotated as alkaloids and 6 unknown peaks with negative correlation were annotated as flavonoids. The exact mass of some non-annotated peaks were similar to those of the predicted intermediates in camptothecin biosynthesis. These results suggest that most of the peaks that positively correlated with $T D C$ expression could be intermediates in camptothecin biosynthesis. We are also aware that if the biosynthetic reactions take place in a protein complex or 'metabolon' with unstable intermediates, it would be difficult to detect and see the correlations the levels of those metabolites with gene expression. 


\section{Experimental}

\subsection{Plant materials}

Sterile $O$. pumila plants were cultured and maintained at $25^{\circ} \mathrm{C}$ with a photoperiod of 18-h light (2000 lux.)/6-h darkness, as described previously (Kitajima et al., 1997).

\subsection{Induction and culture of callus}

One-centimeter fragments of 3-week-old hairy roots of $O$. pumila (Saito et al., 2001) were cultured on B5 medium (Gamborg et al., 1968) containing 2\% sucrose, $0.2 \%$ gellan gum, $0.5 \mu \mathrm{M}$ 1-naphthaleneacetic acid (NAA), and $5 \mu \mathrm{M} \mathrm{N} \mathrm{N}^{6}$-benzyladenine (BA) at $25^{\circ} \mathrm{C}$ under dark conditions. After 3 weeks, a yellowish-white callus was induced from the hairy root fragments. The induced callus was maintained by subculturing once a month under the same conditions. Cell suspension cultures were established by culturing the callus in B5 liquid medium containing $2 \%$ sucrose, $0.5 \mu \mathrm{M}$ $\mathrm{NAA}$, and $5 \mu \mathrm{M} \mathrm{BA}$ at $25^{\circ} \mathrm{C}$ on a rotary shaker $(125 \mathrm{rpm})$ under dark conditions. The established cell suspension cultures were maintained by subculturing every 2 weeks under the same conditions. 


\subsection{Vector construction for RNAi}

To construct RNAi vectors, Gateway ${ }^{\circledR}$ technology (Invitrogen, Carlsbad, CA,

USA) was used. The attB site was incorporated by 2 PCR steps using gene-specific attB primers, according to the manufacturer's instructions. A 468-bp fragment (TDCi fragment) corresponding to the $5^{\prime}$ end of the coding regions of $O$. pumila tryptophan decarboxylase gene (TDC) (Yamazaki et al., 2003b) was cloned into the donor vector pDONR221 using BP clonase. Similarly, a 425-bp fragment (SLSi fragment) without the conserved regions of cytochrome $\mathrm{P} 450$ from $O$. pumila secologanin synthase gene (SLS) (unpublished data) was selected and cloned into pDONR221. The gene-specific $a t t \mathrm{~B}$ primers designed for the TDCi and SLSi fragments are as follows: TDCi-attB1 (AAAAAGCAGGCTATGGGCAGCATTAGTGAAAATTGTGATG) and TDCi-attB2 (AGAAAGCTGGGTACCATGAAACATGAAAGATTTAGGCAACTT) for the TDCi fragment; SLSi-attB1 (AAAAAGCAGGCTGGATGGGACGCATTCCGAGAG) and SLSi-attB2 (AGAAAGCTGGGTCATGGTCTCAATGGTGAGGTCC) for the SLSi fragment. The integrity of the constructs was verified by DNA sequencing. The entry clones containing the TDCi or SLSi fragments were recombined in an LR reaction with pGWB80 of the binary vector system for RNAi and selected with hygromycin. For 
evaluating the RNAi effect, a control vector containing the coding regions of Arabidopsis thaliana $\beta$-glucuronidase (GUS) gene (Kertbundit et al., 1991) was constructed.

\subsection{Induction and culture of RNAi hairy roots}

The plasmids pGWB80-TDCi, pGWB80-SLSi, and pGWB80-GUSi were transferred to Agrobacterium rhizogenes 15834 by electroporation using $0.1-\mathrm{cm}$ cuvettes. The A. rhizogenes 15834 clones carrying these RNAi vectors were grown at $28^{\circ} \mathrm{C}$ in YEB liquid medium for 1 day. After that, they were co-cultured with 1-cm cut stems of young aseptic O. pumila plants (Kitajima et al., 1997) in B5 liquid medium containing $2 \%$ sucrose. After 2 days, the infected stems were washed with fresh B5 liquid medium containing $2 \%$ sucrose, and then, cultured on B5 medium containing $2 \%$ sucrose and $0.2 \%$ gellan gum supplemented with $200 \mathrm{mg} \cdot \mathrm{L}^{-1}$ of cefotaxime $\left(\right.$ Claforan ${ }^{\circledR}$ ) for disinfection at $25^{\circ} \mathrm{C}$ under dark conditions. After 3 weeks, the hairy roots that emerged from the stem fragments were excised and cultured on B5 medium containing $2 \%$ sucrose and $0.2 \%$ gellan gum supplemented with $200 \mathrm{mg} \cdot \mathrm{L}^{-1}$ of cefotaxime and 25 $\mathrm{mg} \cdot \mathrm{L}^{-1}$ of hygromycin for disinfection and selection at $25^{\circ} \mathrm{C}$ under dark conditions. The established RNAi hairy roots, TDCi-HR, SLSi-HR, and GUSi-HR, were subcultured 
once a month on B5 medium containing $2 \%$ sucrose and $0.2 \%$ gellan gum. For gene expression and metabolite analyses, RNAi hairy roots were cultured in B5 liquid medium containing $2 \%$ sucrose for 3 weeks at $25^{\circ} \mathrm{C}$ on a rotary shaker $(80 \mathrm{rpm})$ under dark conditions.

\subsection{Gene expression analysis}

Total RNA was isolated from 3-week-old RNAi hairy roots using RNeasy plant mini kit (Qiagen, Hilden, Germany). Reverse transcription was carried out at $42^{\circ} \mathrm{C}$ in a $20-\mu \mathrm{L}$ solution containing $1 \mu \mathrm{g}$ of total RNA, $0.5 \mu \mathrm{g}$ of Oligo(dT) ${ }_{12-18}$ primer (Invitrogen), $10 \mathrm{nmol}$ of dNTPs (Takara Bio, Shiga, Japan), $0.2 \mu \mathrm{mol}$ of DTT (Invitrogen), and 200 units of SuperScript II Reverse Transcriptase (Invitrogen). First-strand cDNA synthesized from $1 \mathrm{ng}$ of total RNA was used for amplification of $T D C$ and the control $\beta$-tubulin gene (TUB) (unpublished data). For semi-quantitative RT-PCR, a 24-cycle PCR reaction for $T D C$ and $S L S$ and a 25-cycle PCR reaction for TUB were carried out with ExTaq DNA polymerase (Takara Bio) and gene-specific primers. The designed gene-specific primers were as follows: TDC-F (ATGGGCAGCATTAGTGAAAA) and TDC-R (TTACTCAATGATATTGGTTTTCGT) for TDC; SLS-F 
(CCAAAGTTGCCTTTGGTAGC) and SLS-R (CAACCCCATCAGCAAATCTT) for

SLS; TUB-F (CCAGATAACTTTGTTTTCGG) and TUB-R

(GTGAACTCCATTTCATCCAT) for TUB. PCR products were separated on 1\% agarose gels and stained with SYBR Green I nucleic acid gel stain (Invitrogen) for 30 min in the dark. The stained gels were washed with distilled water for $10 \mathrm{~min}$ in the dark and scanned with Storm 860 (GE Healthcare, Little Chalfont, Buckinghamshire, England) using the following parameters: blue fluorescence $(450 \mathrm{~nm})$ and photomultiplier tube (PMT) gain of $850 \mathrm{~V}$. The scanned images were visualized and analyzed using Image Quant (GE Healthcare), according to the manufacturer's protocols.

\subsection{Analysis of camptothecin-related alkaloids}

For alkaloid extraction, 3-week-old hairy roots and cell suspension cultures were completely dried in a lyophilizer. They were disrupted using a Multi-bead shocker (Yasui Kikai, Osaka, Japan), and $1 \mathrm{~mL}$ of methanol was added per $10 \mathrm{mg}$ of dried tissue. The mixture was sonicated for $30 \mathrm{~min}$ and the homogenates were stored overnight at $4^{\circ} \mathrm{C}$. On the next day, the homogenates were transferred to microtubes and centrifuged at $10,000 \mathrm{~g}$ for $10 \mathrm{~min}$. The clear supernatants were transferred to new microtubes and 
100-fold dilution of supernatants were applied on reverse-phase HPLC using a TSK gel ODS-80TM column $(4.6 \mathrm{~mm} \times 150 \mathrm{~mm}$; TOSOH, Tokyo, Japan) with a solvent system of methanol: $\mathrm{H}_{2} \mathrm{O}(7: 3)$. Camptothecin was monitored by its characteristic fluorescence (excitation at $365 \mathrm{~nm}$ and emission at $428 \mathrm{~nm}$ ) (Asano et al., 2004).

For the profiling of camptothecin-related alkaloids, the methanol extracts were analyzed by HPLC/DAD/ESI/MS, as described previously (Asano et al., 2004; Yamazaki et al., 2003a).

\subsection{Non-targeted metabolite analysis using infusion FTICR-MS}

High-, middle- and non-polar extracts of plant materials were subjected to FT-MS (APEX III FT-ICMS; Bruker Daltonics, Billerica, MA, USA), as described previously (Aharoni et al., 2002; Hirai et al., 2004, Tohge et al., 2005). The fold change in each mass peak was calculated as the ratio of the signal intensity in hairy roots of $O$. pumila to that in cell suspension culture induced from hairy roots. Data mining of metabolites was based on elemental composition calculated from an accurate $\mathrm{m} / z$ value using DISCOVArray (Phenomenome Discoveries, Saskatoon, SK, Canada).

\subsection{Semi-quantification analysis using LC-FTICR-MS}


For LC-FTICR-MS analysis, Agilent 1100 system (Agilent, Santa Clara, CA, USA) coupled to Finnigan LTQ-FT (Thermo Fisher Scientific, Waltham, MA, USA) and Xcalibur software version 2.0 (Thermo Fisher Scientific) was used (Iijima et al., 2008, Suzuki et al., 2008). For HPLC separation, Mightysil-RP18-GP $(4.6 \mathrm{~mm} \times 250$ $\mathrm{mm}, 5 \mu \mathrm{m}$; Kanto Chemical, Tokyo, Japan) was used at a flow rate of $0.6 \mathrm{~mL} / \mathrm{min}$. A combination of pure water and $0.25 \% \mathrm{v} / \mathrm{v}$ acetic acid and methanol was used as the mobile phase. Mass spectra were acquired according to a previously described method (Iijima et al., 2008). 


\section{Acknowledgments}

We thank Tsuyoshi Nakagawa (Shimane University) for providing the

pGWB80 vector, Ushio Sankawa (Toyama International Research Center for Traditional Medicine) and Yasuyo Yamazaki (Phenomenome Discoveries) for infusion FTICR-MS analysis, and Norio Aimi, Hiromitsu Takayama and Mariko Kitajima (Chiba University) for providing the isolated compounds from $O$. pumila and useful suggestions throughout the study. This work was supported in part by Grant-in-Aid for Scientific Research on Innovative Areas from The Ministry of Education, Culture, Sports, Science and Technology (MEXT), and by CREST of the Japan Science and Technology Agency (JST). 


\section{Figure legends}

Fig. 1. Predicted pathway for camptothecin biosynthesis from tryptophan and geraniol in O. pumila

Enzyme abbreviations: TDC, tryptophan decarboxylase; G10H, geraniol 10-hydroxylase; SLS, secologanin synthase; STR, strictosidine synthase.

Fig. 2. HPLC chromatograms of $O$. pumila cell cultures

(A) Hairy root cultures (B) cell suspension cultures. The peak numbers correspond to the following compounds: 1, camptothecin; 2, pumiloside; 3, 3-O-caffeoylquinic acid; 4, Lucidin 3-O- $\beta$-purimeveroside. The HPLC conditions were as follows: column, Mightysil RP-18 $(4.6 \times 250 \mathrm{~mm})$; gradient, linear gradient from solvent A to solvent B ( $0-35 \mathrm{~min})$, isocratic at $100 \%$ of solvent B ( $35-40 \mathrm{~min})$; solvent A ( $20 \%$ methanol, $0.2 \%$ acetic acid in $\mathrm{H}_{2} \mathrm{O}$ ), solvent $\mathrm{B}\left(90 \%\right.$ methanol, $0.025 \%$ acetic acid in $\mathrm{H}_{2} \mathrm{O}$ ); flow rate, $0.8 \mathrm{~mL} \cdot \mathrm{min}^{-1}$; column temperature, $37^{\circ} \mathrm{C}$.

Fig. 3. Correlation analyses between the expression of targeted genes and accumulation of camptothecin in RNAi hairy roots 
The relative expression level was calculated with respect to the average expression level of the targeted genes in GUSi-HR. (A) TDCi-HR and GUSi-HR, and (B) SLSi-HR and GUSi-HR. Bars: Standard deviation ( $\mathrm{n}=3$ per analysis).

Fig. 4. HPLC chromatograms of RNAi hairy roots of $O$. pumila

(A) TDCi-HR and GUSi-HR, and (B) SLSi-HR and GUSi-HR. The HPLC conditions were as described in Fig. 2.

Fig. 5. Scheme for the selection of new candidates for intermediates in camptothecin biosynthesis

Fig. 6. Correlation analyses between expression of targeted genes and accumulation of camptothecin-related alkaloids in RNAi hairy roots

The relative expression level was calculated with respect to the average expression levels of the targeted genes in GUSi-HR $(n=1)$.

Fig. 7. Correlation analyses between expression of targeted genes and accumulation of secologanin and its intermediates in RNAi hairy roots 
The relative expression level was calculated with respect to the average expression levels of the targeted genes in GUSi-HR $(n=1)$

Fig. 8. Secologanin biosynthetic pathway

Fig. 9. Possible post-strictosidine pathway and intermediates in camptothecin biosynthesis

Ex Ms is the exact mass calculated from the molecular formula. 


\section{References}

Afendi, F.M., Okada, T., Yamazaki, M., Morita, A.H., Nakamura, Y., Nakamura, K., Ikeda, S., Takahashi, H., Alatuf-Ul-Amin, M., Darusman, L.K., Saito, K., Kanaya, S., in press. KNApSAcK Family Databases: Integrated metabolite-plant species databases for multifaceted plant researches. Plant Cell Physiol.

Aharoni, A., De Vos, R.C.H., Verhoeven, H.A., Maliepaard, C.A., Kruppa, G., Bino, R., Goodenowe, D.B., 2002. Non-targeted metabolome analysis by use of Fourier transform ion cyclotron mass spectrometry. OMICS 6, 217-243.

Aimi, N., Nishimura, M., Miwa, A., Hoshino, H., Sakai, S., Haginiwa, J., 1989. Pumiloside and deoxypumiloside; plausible intermediates of camptothecin biosynthesis. Tetrahedron Lett. 30, 4991-4994.

Aimi, N., Seki, H., Sakai, S., 1992. Synthesis of lyaloside, a prototypal $\beta$-carboline gluco indole alkaloid in Rubiaceous plants. Chem. Pharm. Bull. 40, 2588-2590.

Asano, T., Watase, I., Sudo, H., Kitajima, M., Takayama, H., Aimi, N., Yamazaki, M., Saito, K., 2004. Camptothecin production by in vitro cultures of Ophiorrhiza liukiuensis and O. kuroiwai. Plant Biotech. 21, 275-281.

Carte, B.K., DeBrosse, C., Eggleston, D., Hemling, M., Mentzer, M., Poehland, B., Troupe, N., Westley, J.W., Hecht, S.M., 1990. Isolation and characterization of a presumed biosynthetic precursor of camptothecin from extracts of Camptotheca acuminata. Tetrahedron 46, 2747-2760.

De Luca, V., Marineau, C., Brisson, N., 1989. Molecular cloning and analysis of cDNA encoding a plant tryptophan decarboxylase: comparison with animal dopa decarboxylases. Proc. Natl. Acad. Sci. USA, 86, 2582-2586. 
Facchini, P.J., De Luca, V., 2008. Opium poppy and Madagascar periwinkle: model non-model systems to investigate alkaloid biosynthesis in plants. Plant J. 54, $763-784$.

Gamborg, O.L., Miller, R.A., Ojima, K., 1968. Nutrient requirements of suspension cultures of soybean root cells. Exp. Cell Res. 50, 151-158.

Govindachari, T.R., Viswanathan, N, 1972. Alkaloids of Mappia foetida. Phytochemistry 11, 3529-3531.

Hirai, M.Y., Yano, M., Goodenowe, B.G., Kanaya, S., Kimura, T., Awazuhara, M., Arita, M., Fujiwara, T., Saito, K., 2004. Integration of transcriptomics and metabolomics for understanding of global responses to nutritional stress in Arabidopsis thaliana. Proc. Natl Acad. Sci. USA 101, 10205-10210.

Hsiang, Y.H., Hertzberg, R., Hecht, S., Liu, L.F., 1985. Camptothecin induces proteinlinked DNA breaks via mammalian DNA topoisomerase I. J. Biol. Chem. 260, 14873-14878.

Hutchinson, C.R., Heckendorf, A.H., Straughn, J.L., Daddona, P.E., in part, Cane, D.E., 1979. Biosynthesis of camptothecin. 3. Definition of strictosamide as the penultimate biosynthetic precursor assisted by ${ }^{13} \mathrm{C}$ and ${ }^{2} \mathrm{H}$ NMR spectroscopy. J. Amer. Chem. Soc. 101, 3358-3369.

Iijima, Y., Nakamura, Y., Ogata, Y., Tanaka, Ken'ichi, Sakurai, N., Suda, K., Suzuki, T., Suzuki, H., Okazaki, K., Kitayama, M, Kanaya, S., Aoki, K., Shibata, D., 2008. Metabolite annotations based on the integration of mass spectral information. Plant J. $54,949-962$.

Irmler, S., Schröder, G., St-Pierre, B., Crouch, N.P., Hotze, M., Schmidt, J., Strack, D., Matern, U., Schröder, J., 2000. Indole alkaloid biosynthesis in Catharanthus roseus: 
new enzyme activities and identification of cytochrome P450 CYP72A1 as secologanin synthase. Plant J. 24, 797-804.

Kertbundit, S., Greve, H.D., Deboeck, F., Montagu, M.V., Hernalsteens, J.P., 1991. In vivo random $\beta$-glucuronidase gene fusions in Arabidopsis thaliana. Proc. Natl. Acad. Sci. USA $88,5212-5216$

Kitajima, M., Nakamura, M., Takayama, H., Saito, K., Stöckigt, J., Aimi, N., 1997. Constituents of regenerated plants of Ophiorrhiza pumila; formation of a new glycocamptothecin and predominant formation of ( $3 R$ )-deoxypumiloside over (3S)-congener. Tetrahedron Lett. 38, 8997-9000.

Kitajima, M., Fischer, U., Nakamura, M., Ohsawa, M., Ueno, M., Takayama, H., Unger, M., Stöckigt, J., Aimi, N., 1998. Anthraquinones from Ophiorrhiza pumila tissue and cell cultures. Phytochemistry 48, 107-111.

Kitajima, M., Yoshida, S., Yamagata, K., Nakamura, M., Takayama, H., Saito, K., Seki, H., Aimi, N., 2002. Camptothecin-related alkaloids from hairy roots of Ophiorrhiza pumila. Tetrahedron 58, 9169-9178.

Kitajima, M., Fujii, N., Yoshino, F., Sudo, H., Saito, K., Aimi, N., Takayama, H., 2005. Camptothecin and two new monoterpene glucosides from Ophiorrhiza liukiuensis. Chem. Pharm. Bull. 53, 1355-1358.

Kitajima, M., Nakamura, T., Kogure, N., Ogawa, M., Mitsuno, Y., Ono, K., Yano, S., Aimi, N., Takayama, H., 2006. Isolation of gelsedine-type indole alkaloids from Gelsemium elegans and evaluation of the cytotoxic activity of Gelsemium alkaloids for A431 epidermoid carcinoma cells. J. Nat. Prod. 69, 715-718.

Kutchan, T.M., 1995. Alkaloid biosynthesis: the basis for metabolic engineering of medicinal plants. Plant Cell 7, 1059-1070. 
Kutchan, T.M., Hampp, N., Lottspeich, F., Beyreuther, K., Zenk, M.H., 1988. The cDNA clone for strictosidine synthase from Rauvolfia serpentina DNA sequence determination and expression in Escherichia coli. FEBS Lett. 237, 40-44.

Morgan, J.A, Shanks, J.V., 2000. Determination of metabolic rate-limitations by precursor feeding in Catharanthus roseus hairy root cultures. J. Biotechnol. 79, $137-145$.

Noé, W., Mollenschott, C., Berlin, J., 1984. Tryptophan decarboxylase from Catharanthus roseus cell suspension cultures: purification, molecular and kinetic data of the homogenous protein. Plant Mol. Biol. 3, 281-288.

Oksman-Caldentey, K.M., Saito, K., 2005. Integrating genomics and metabolomics for engineering plant metabolic pathways. Curr. Opin. Biotech. 16, 174-179.

Pizzolato, J.F., Saltz, L.B., 2003. The camptothecins. Lancet 361, 2235-2242.

Przybylska, M., Marion, L., 1961. The molecular structure of (-)-N-methyl-gelsemicine hydriodide, $\mathrm{C}_{21} \mathrm{H}_{29} \mathrm{O}_{4} \mathrm{~N}_{2}$ I. Can. J. Chem. 39, 2124-2127.

Saito, K., Sudo, H., Yamazaki, M., Koseki-Nakamura, M., Kitajima, M., Takayama, H., Aimi, N., 2001. Feasible production of camptothecin by hairy root culture of Ophiorrhiza pumila. Plant Cell Rep. 20, 267-271.

Sirikantaramas, S., Asano, T., Sudo, H., Yamazaki, M., Saito, K., 2007. Camptothecin: Therapeutic potential and biotechnology. Curr. Pharm. Biotech., 8, 196-202.

Stöckigt, J., Zenk, M.H., 1977. Strictosidine (isovincoside): the key intermediate in the biosynthesis of monoterpenoid indole alkaloids. J. Chem. Soc. Chem. Commun. 18, $646-648$.

Suzuki, H., Sasaki, R., Ogata, Y., Nakamura, Y., Sakurai, N., Kitajima, M., Takayama, H., Kanaya, S., Aoki, K., Shibata, D., Saito, K., 2008. Metabolic profiling of 
flavonoids in Lotus japonicus using liquid chromatography Fourier transform ion cyclotron resonance mass spectrometry. Phytochemistry 69, 99-111.

Tohge, T., Nishiyama, Y., Hirai, M.Y., Yano, M., Nakajima, J., Awazuhara, M., Inoue, E., Takahashi, H., Goodenowe, D.B., Kitayama, M., Noji, M., Yamazaki, M., Saito, K., 2005. Functional genomics by integrated analysis of metabolome and transcriptome of Arabidopsis plants over-expressing an MYB transcription factor. Plant J. 42, $218-235$.

Wall, M.E., Wani, M.C., Cook, C.E., Palmer, K.H., McPhail, A.T., Sim, G.A., 1966. Plant antitumor agents. I. The isolation and structure of camptothecin, a novel alkaloidal leukemia and tumor inhibitor from Camptotheca acuminata. J. Am. Chem. Soc. $88,3888-3890$.

Watase, I., Sudo, H., Yamazaki, M., Saito, K., 2004. Regeneration of transformed Ophiorrhiza pumila plants producing camptothecin. Plant Biotech. 21, 337-342.

Whitmer, S., Canel, C., Hallard, D., Gonçalves, C., Verpoorte, R., 1998. Infuence of precursor availability on alkaloid accumulation by transgenic cell line of Catharanthus roseus. Plant Physiol. 116, 853-857.

Yamamoto, H., Katano, N., Ooi, A. and Inoue, K., 2000. Secologanin synthase which catalyzes the oxidative cleavage of loganin into secologanin is a cytochrome P450. Phytochemistry 53, 7-12.

Yamazaki, Y., Urano, A., Sudo, H., Kitajima, M., Takayama, H., Yamazaki, M., Aimi, N., Saito, K., 2003a. Metabolite profiling of alkaloids and strictosidine synthase activity in camptothecin producing plants. Phytochemistry 62, 461-470.

Yamazaki, Y., Sudo, H., Yamazaki, M., Aimi, N., Saito, K., 2003b. Camptothecin biosynthetic genes in hairy roots of Ophiorrhiza pumila: cloning, characterization 
and differential expression in tissues and by stress compounds. Plant Cell Physiol. 44, 395-403.

Yamazaki, Y., Kitajima, M., Arita, M., Takayama, H., Sudo, H., Yamazaki, M., Aimi, N., Saito, K., 2004. Biosynthesis of camptothecin. In silico and in vivo tracer study from $\left[1-{ }^{13} \mathrm{C}\right]$ glucose. Plant Physiol., 134, 161-170.

Zenk, M.H., Juenger, M., 2007. Evolution and current status of the phytochemistry of nitrogenous compounds. Phytochemistry 68, 2757-2772. 


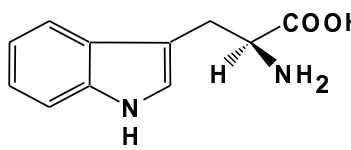

L-Tryptophan

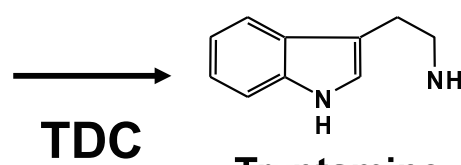

Tryptamine

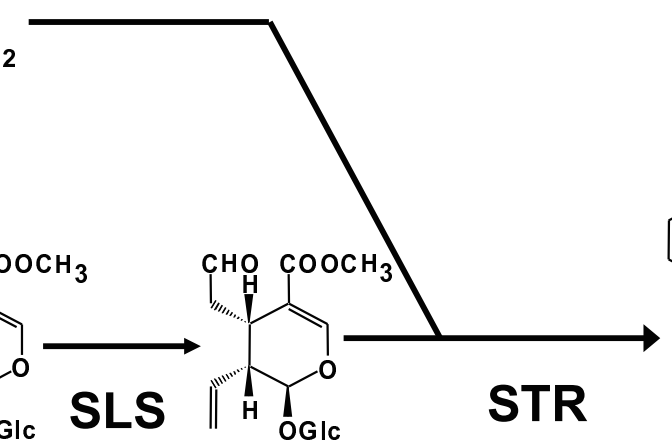

Secologanin

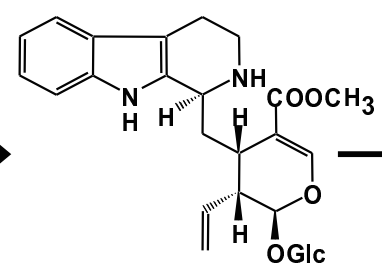

Strictosidine

Geraniol

10-Hydroxygeraniol

Loganin

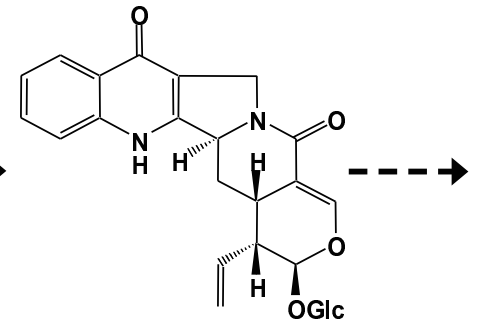

Pumiloside

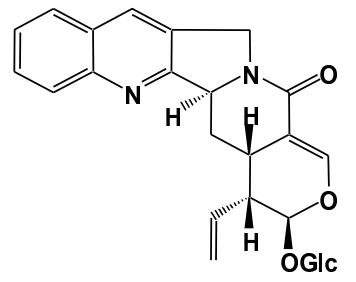

3(S)-Deoxypumiloside

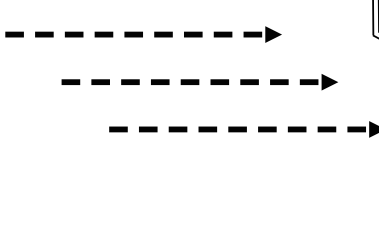

Strictosamide

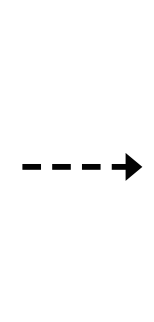

- 
Fig. 2
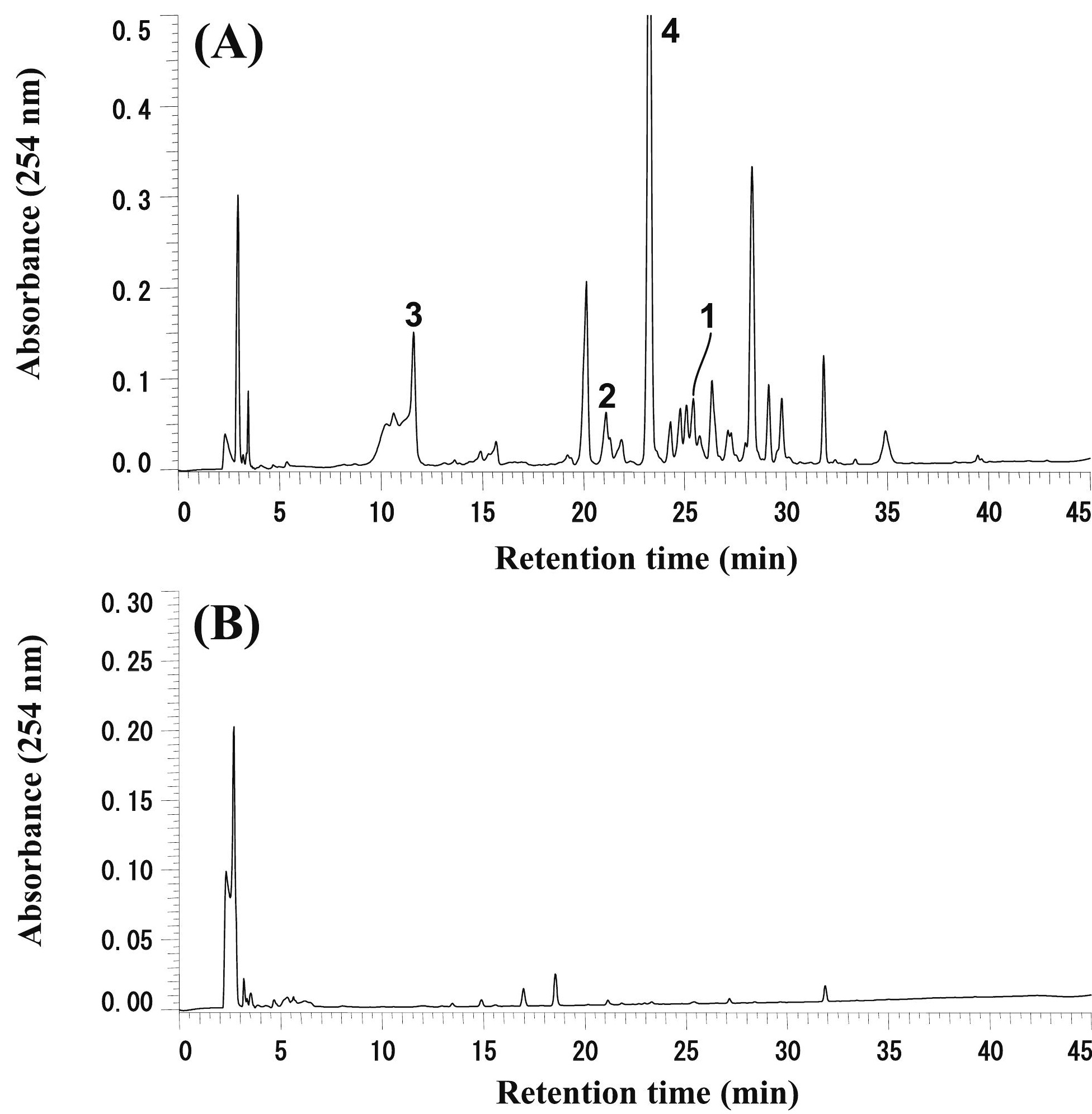
(A)

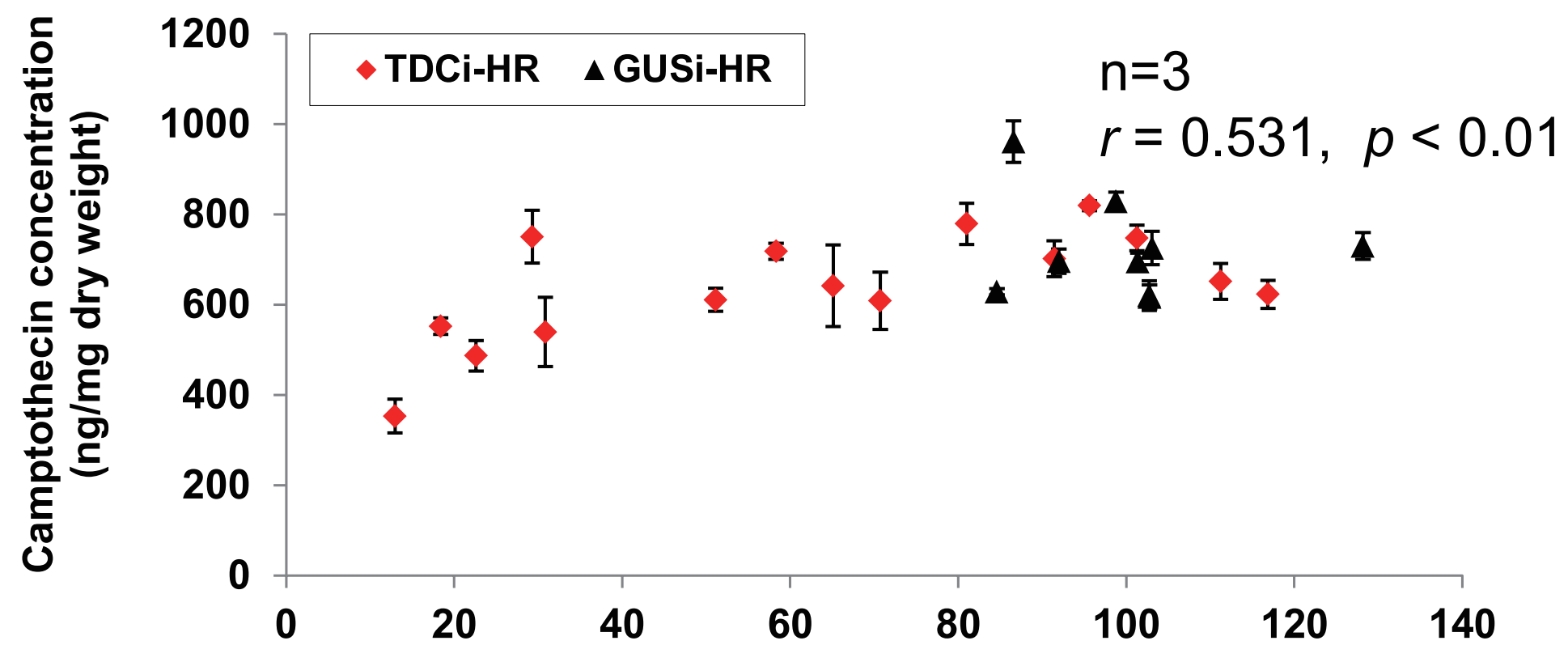

Relative TDC expression (\%)

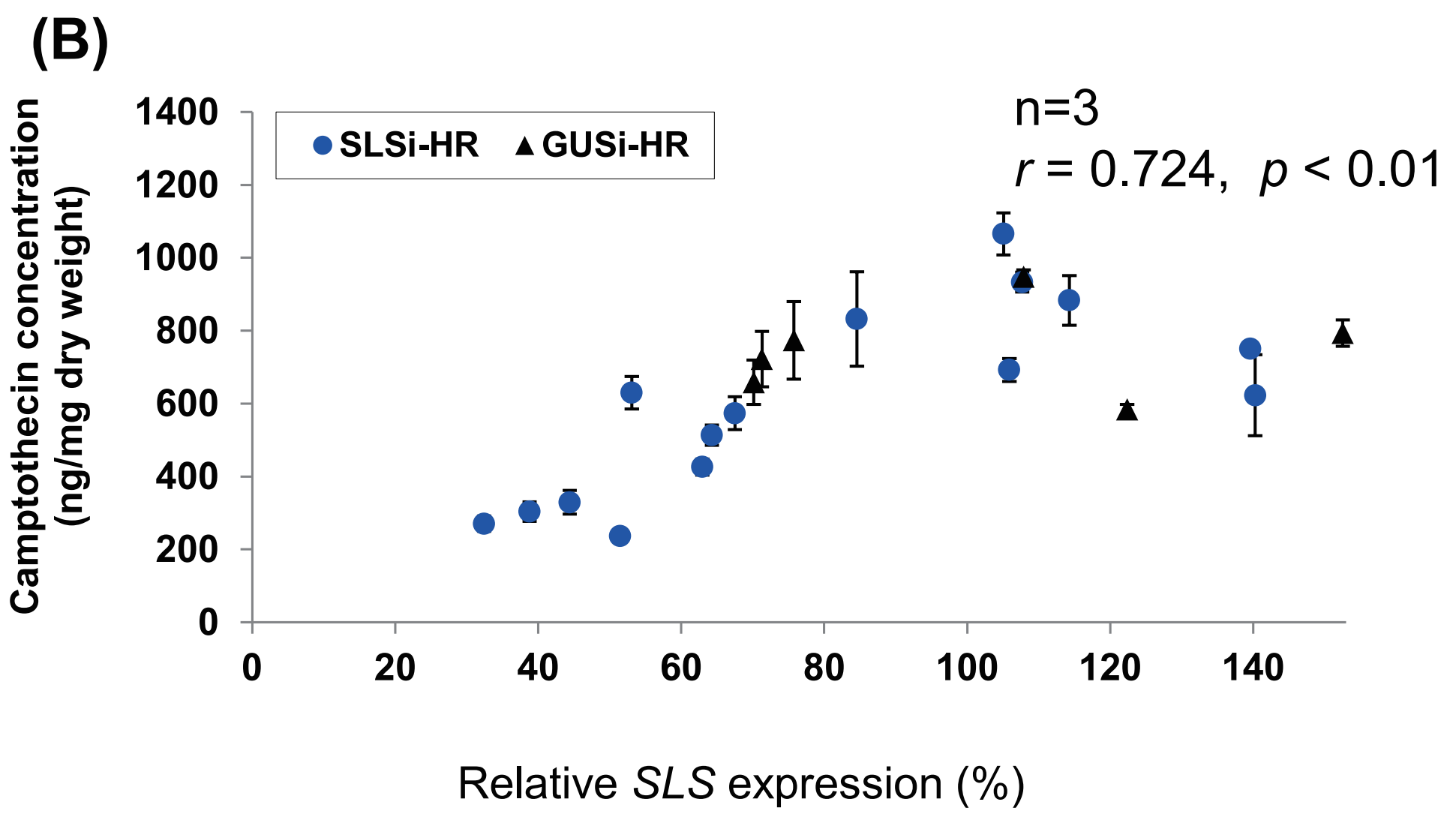




\section{Fig. 4}

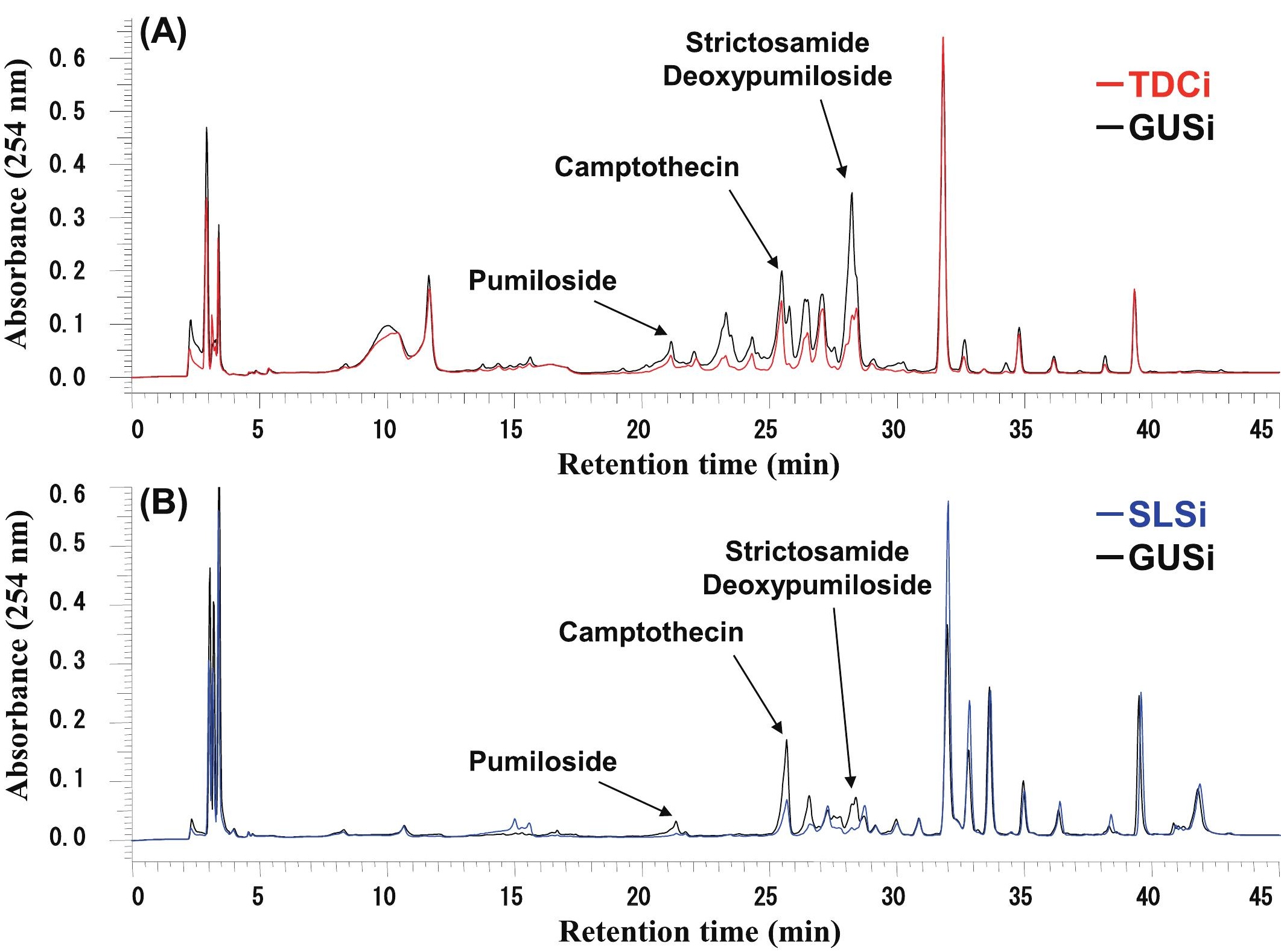




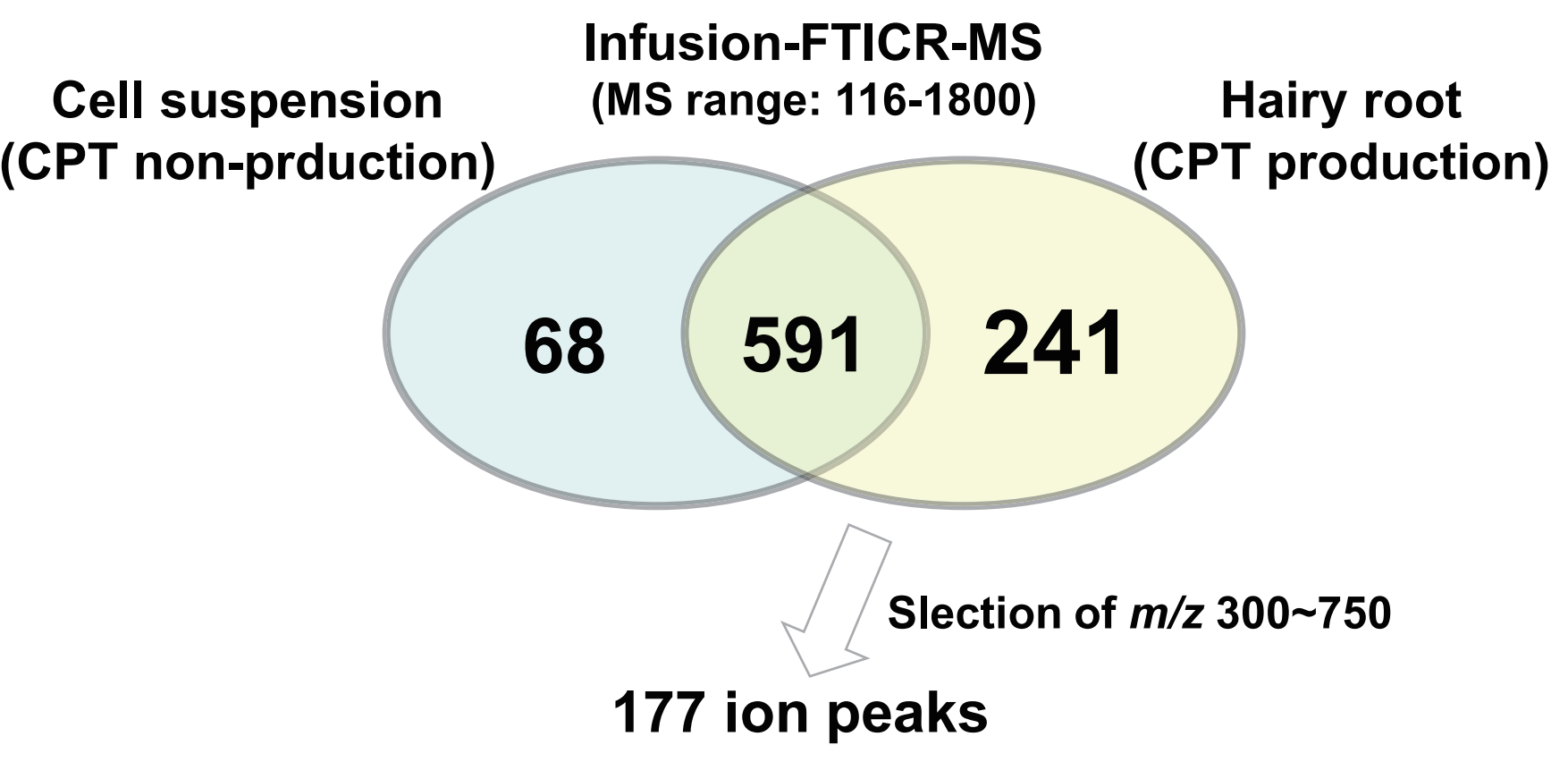

Slection

by LC-FT/MS detection

71 ion peaks

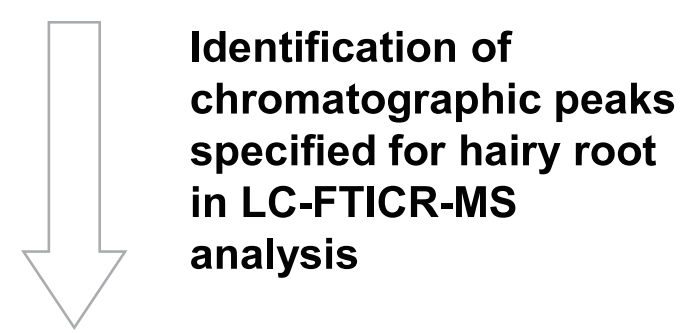

114 chromatographic peaks

Slection of unkno
$\begin{aligned} & \text { Selection by corren } \\ & \text { between TDC expreak } \\ & \text { peak area } \\ & (p<0.1)\end{aligned}$
ve correlation

\section{2 chromatographic peaks with positive correlation}

\section{1 chromatographic peaks with negative correlation}




\section{TDCi-HR $\odot$ SLSi-HR $\Delta$ GUSi-HR}

Camptothecin

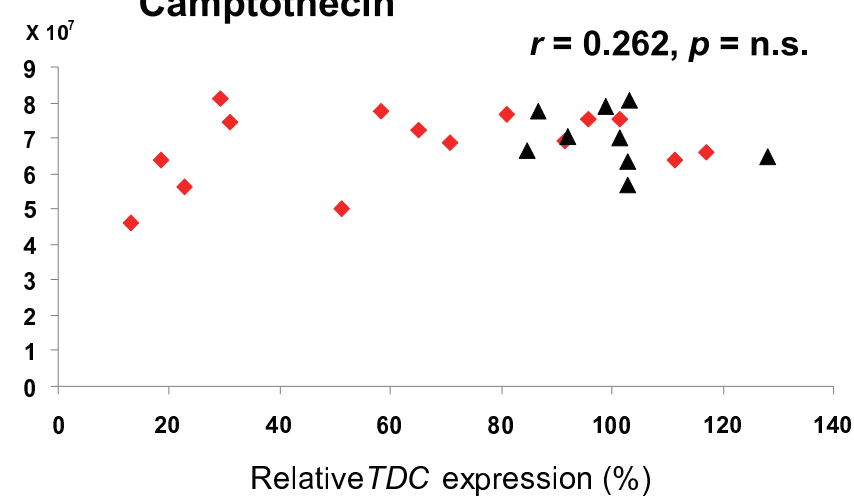

Strictosidine

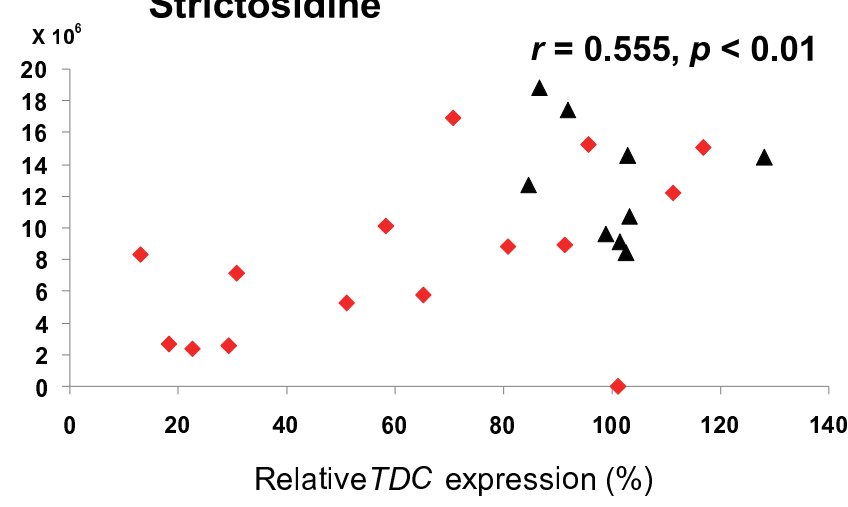

\section{Strictosamide}

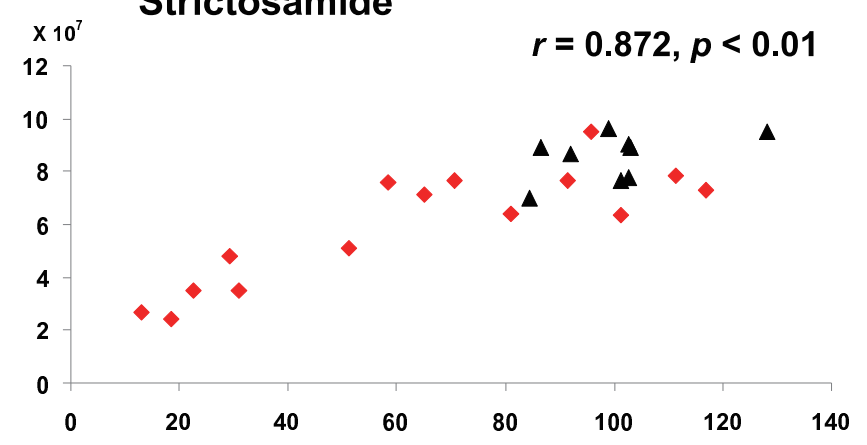

Relative TDC expression (\%)

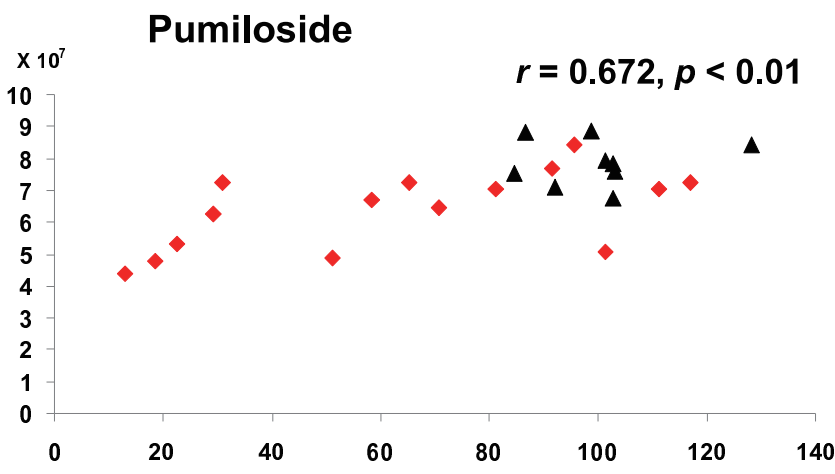

Relative TDC expression (\%)

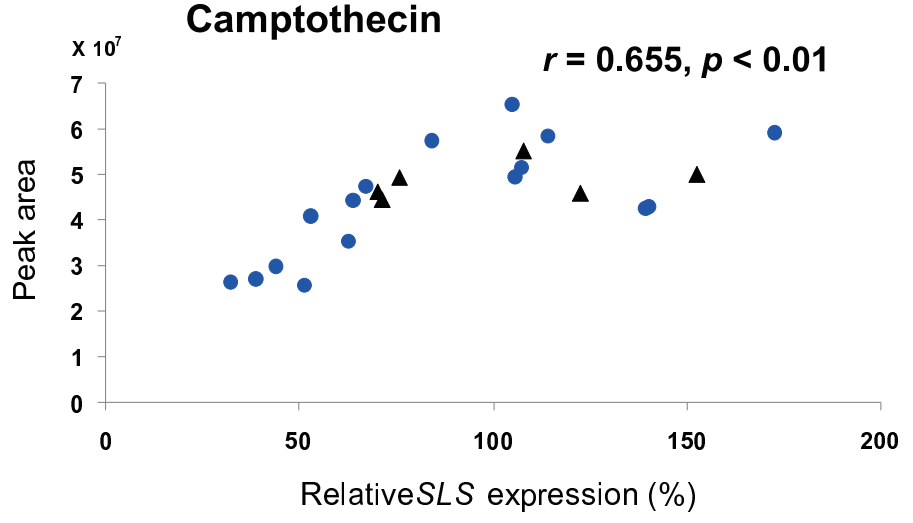

Strictosidine
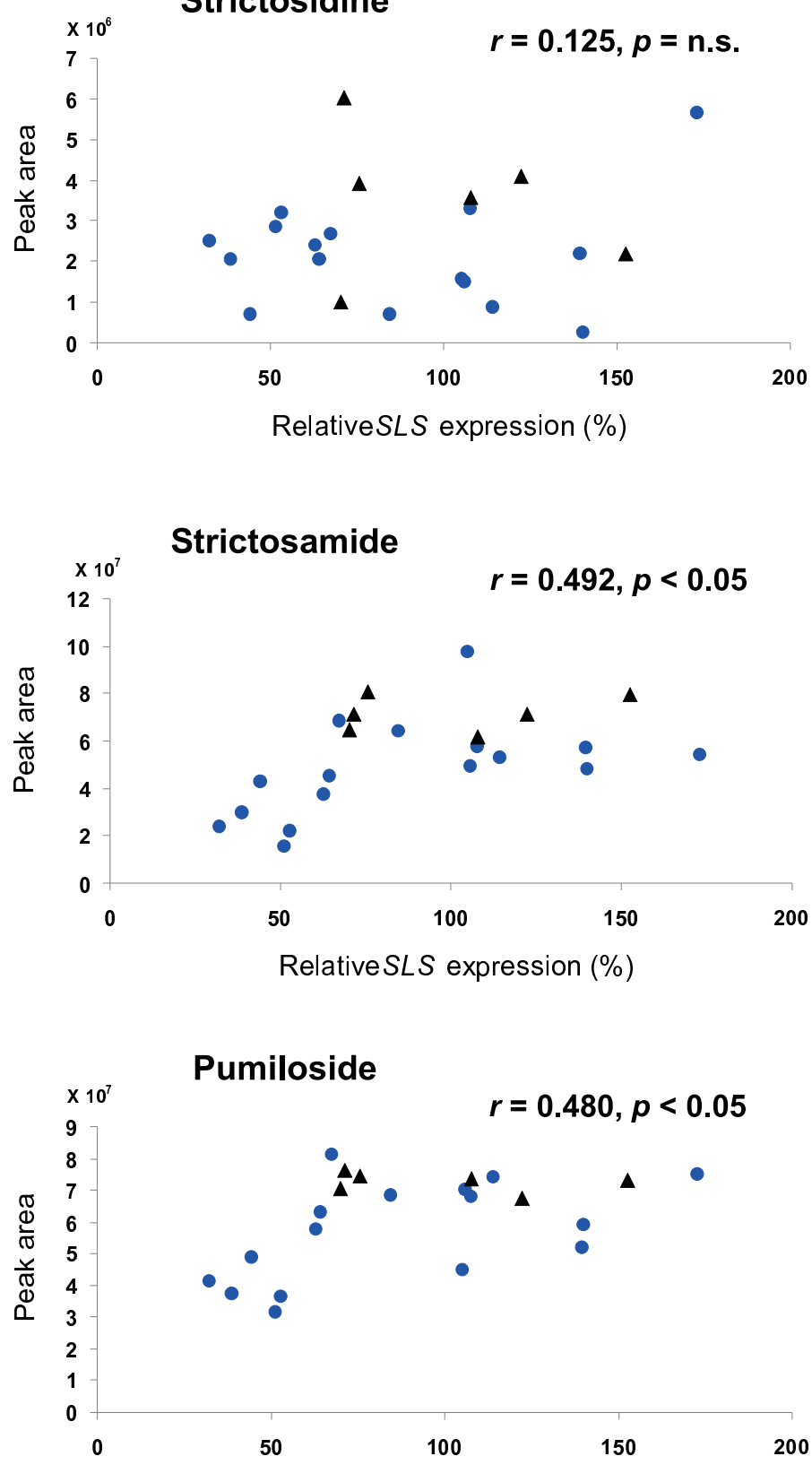

Relative $S L S$ expression (\%) 


\section{Fig. 6 \\ (continued)}

\section{- TDCi-HR • SLSi-HR $\triangle$ GUSi-HR}

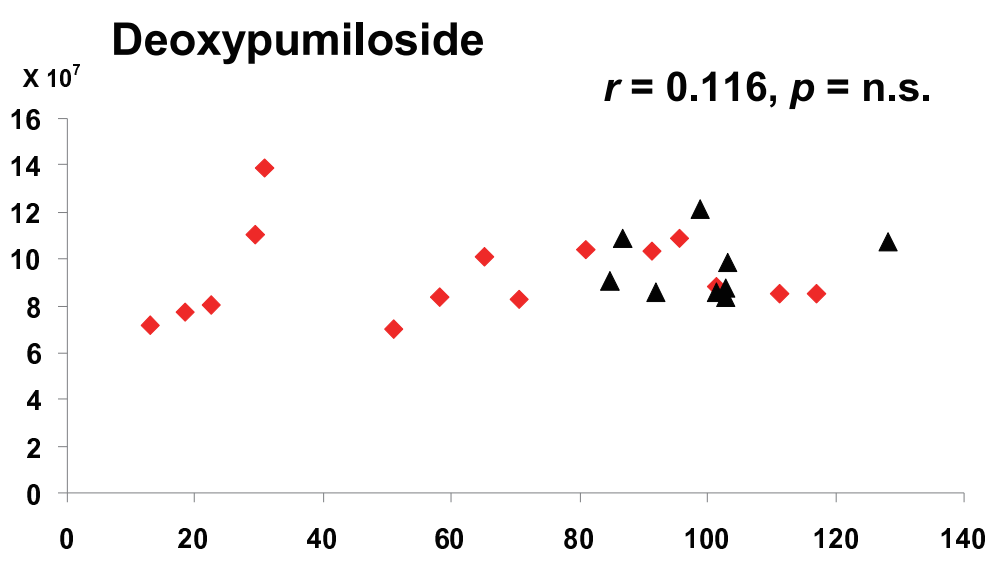

Relative TDC expression (\%)

Pumiloside-like
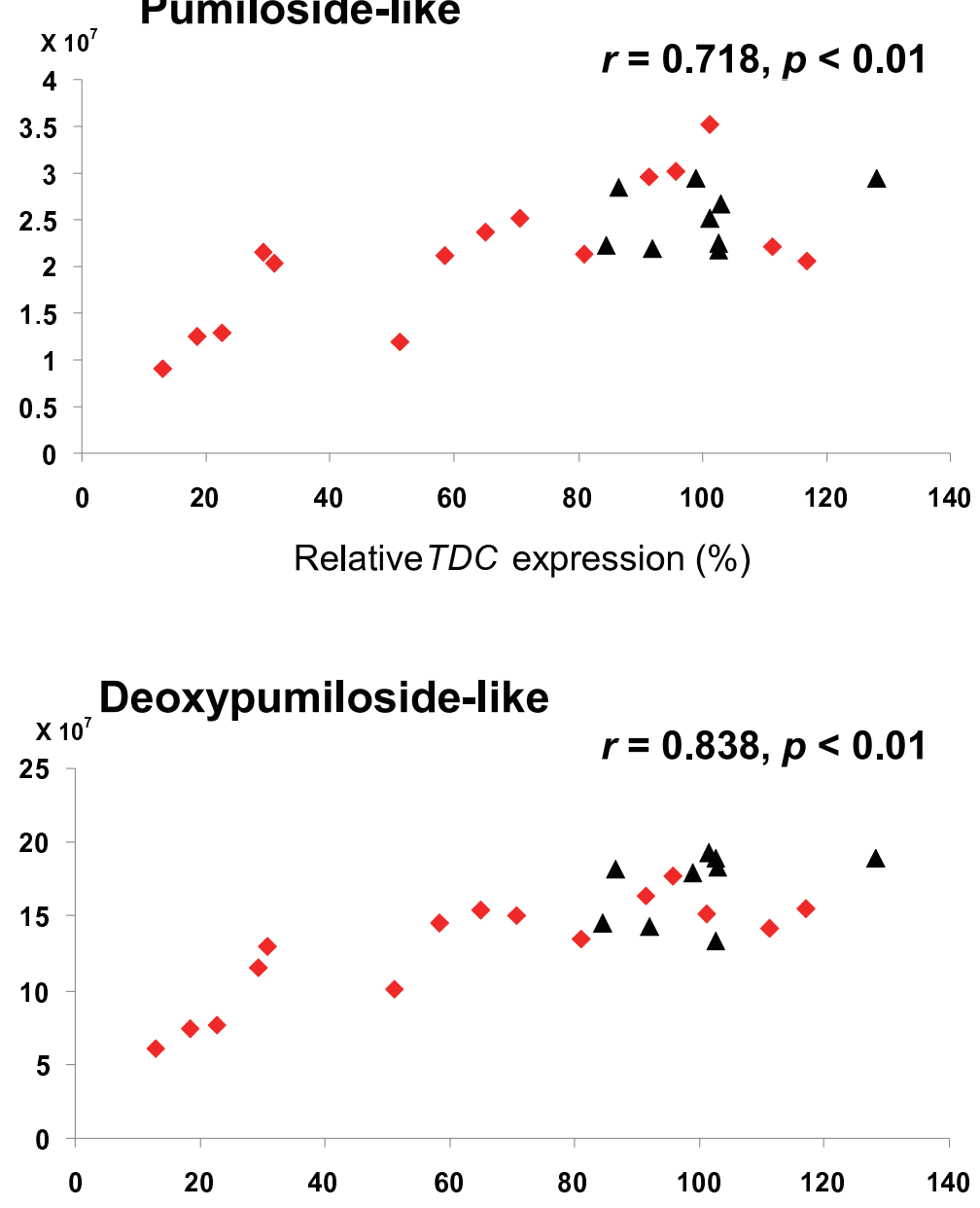

Relative TDC expression (\%)

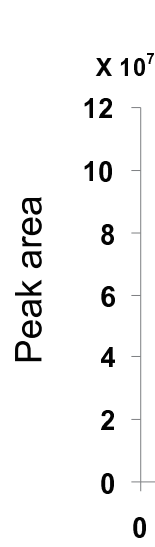

Deoxypumiloside

$r=0.534, p<0.01$

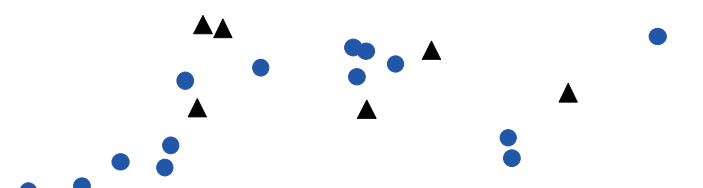

$50 \quad 100$

150

RelativeSLS expression (\%)
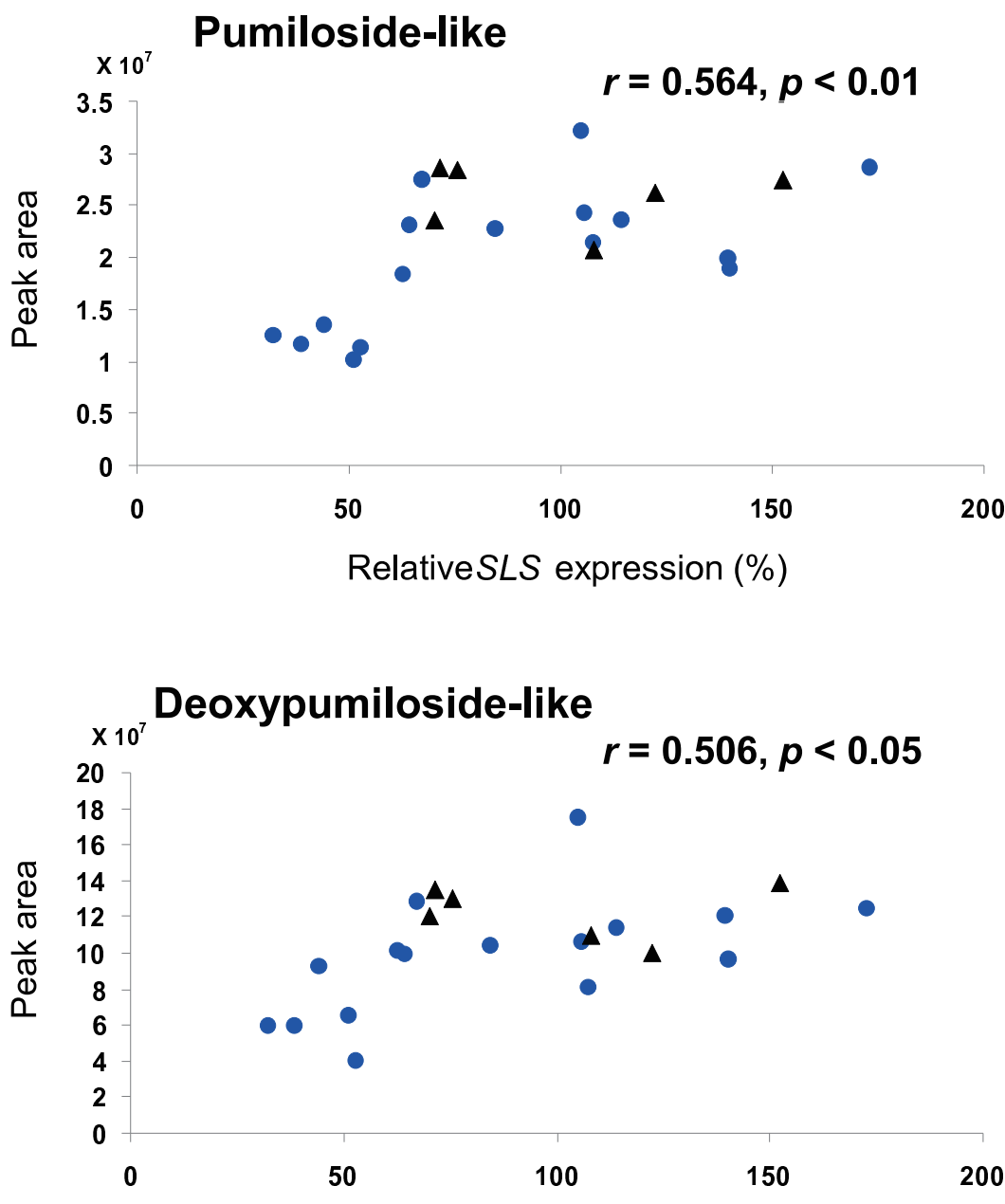


\section{TDCi-HR SLSi-HR $\triangle$ GUSi-HR}
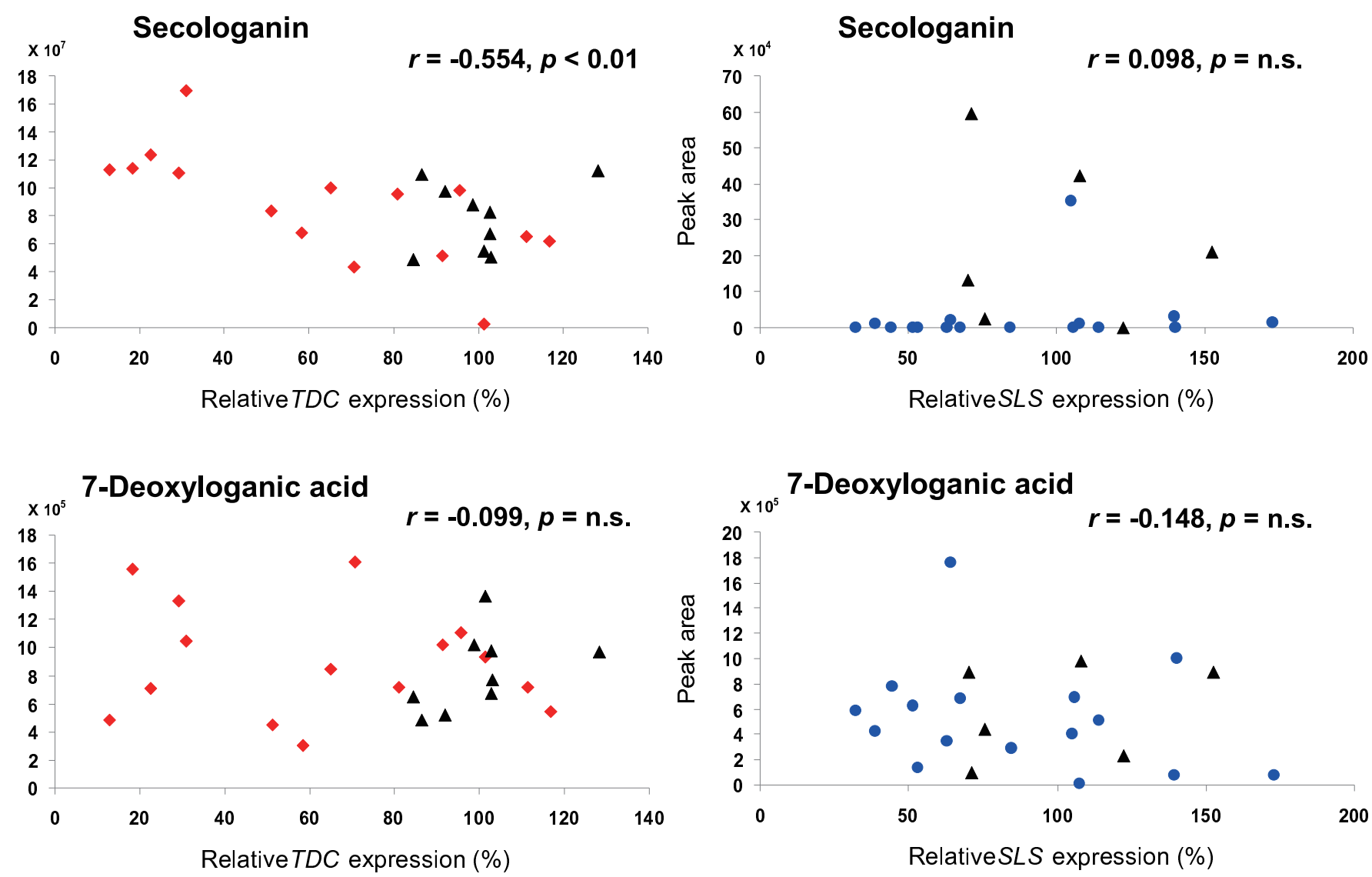

Relative TDC expression (\%)

Loganic acid

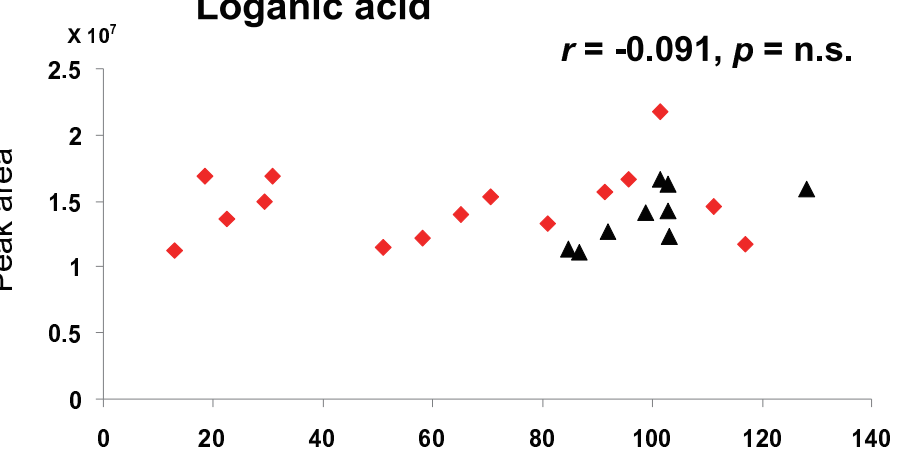

Relative TDC expression (\%)

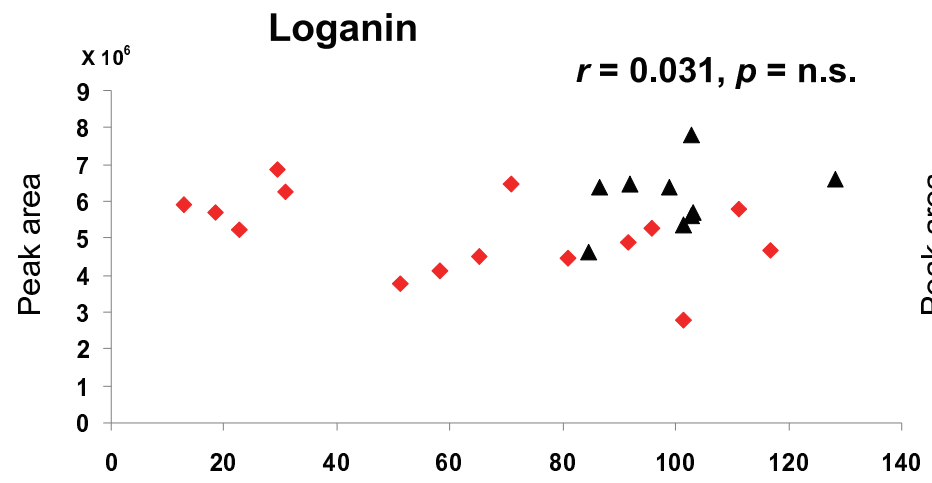

Relative TDC expression (\%)

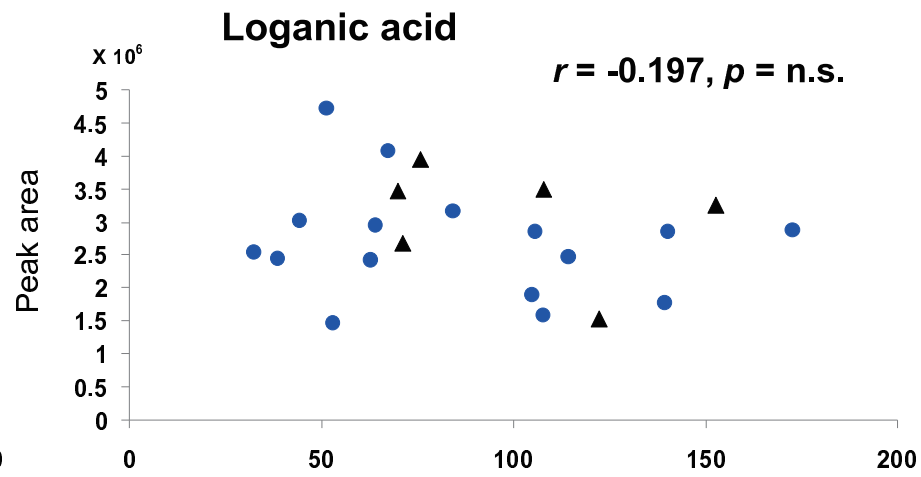

RelativeSLS expression (\%)

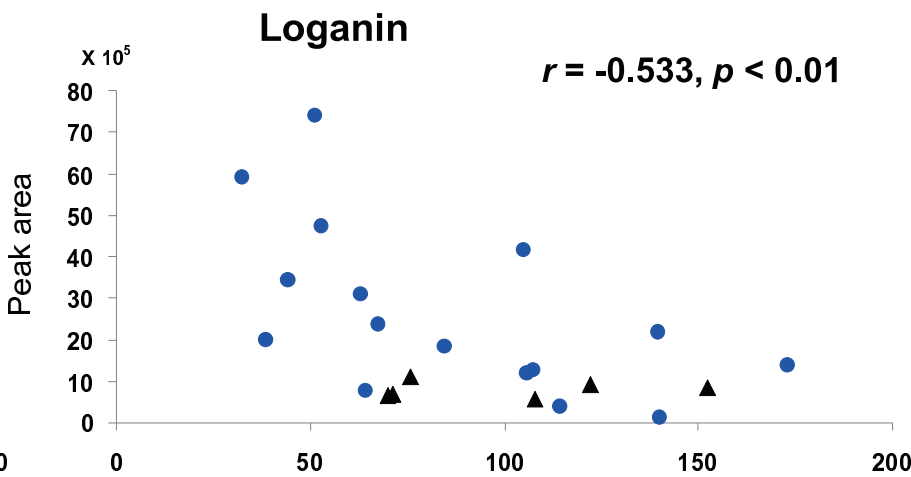

Relative SLS expression (\%) 


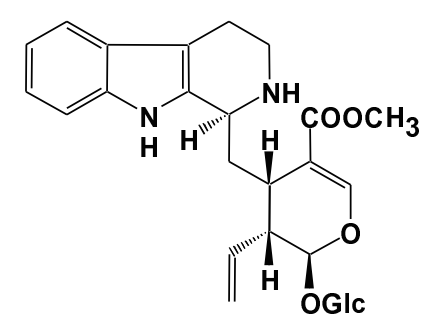

Strictosidine

$\mathrm{C}_{27} \mathrm{H}_{34} \mathrm{~N}_{2} \mathrm{O}_{9}$

Ex MS: 530.2264

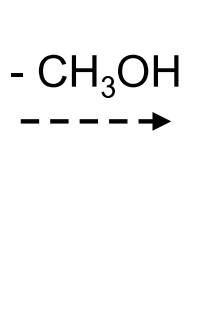

Strictosamide

$\mathrm{C}_{26} \mathrm{H}_{30} \mathrm{~N}_{2} \mathrm{O}_{8}$

Ex MS: 498.2002

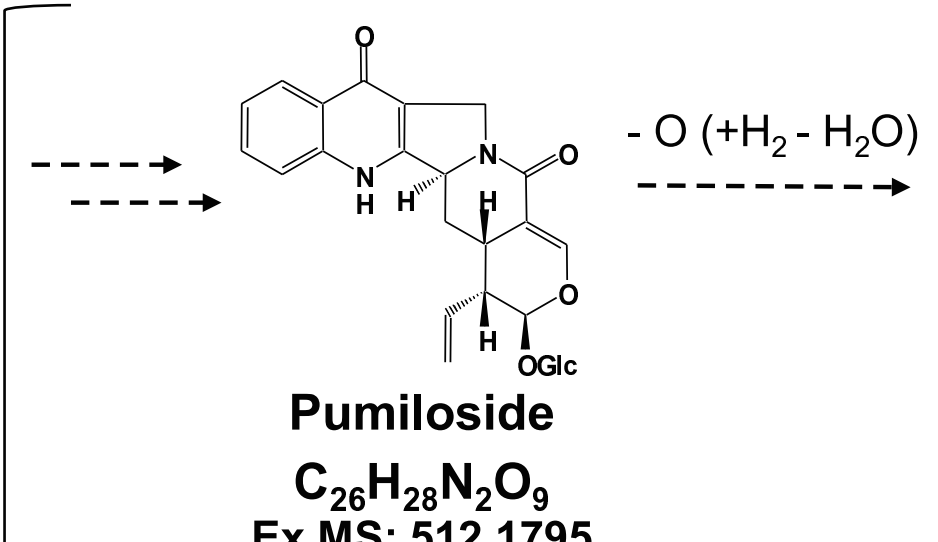

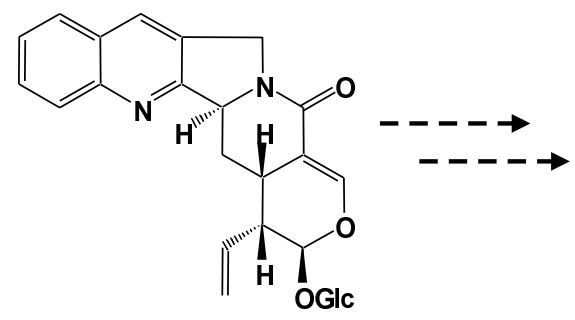

3(S)-Deoxypumiloside $\mathrm{C}_{26} \mathrm{H}_{28} \mathrm{~N}_{2} \mathrm{O}_{8}$ Ex MS: 496.1846

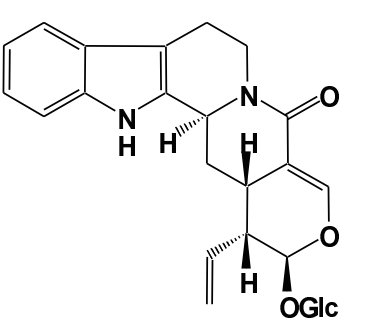

Ex MS: 512.1795
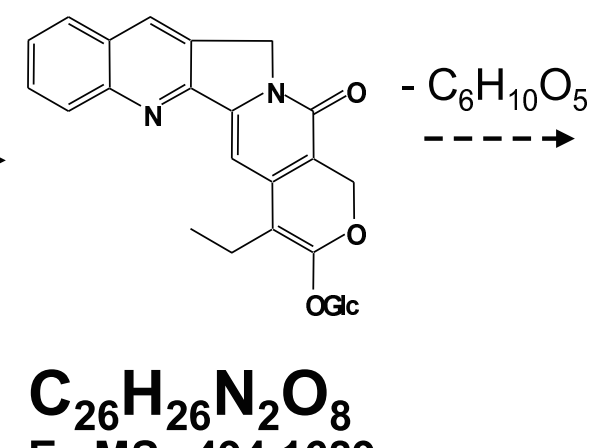

Ex MS: 494.1689

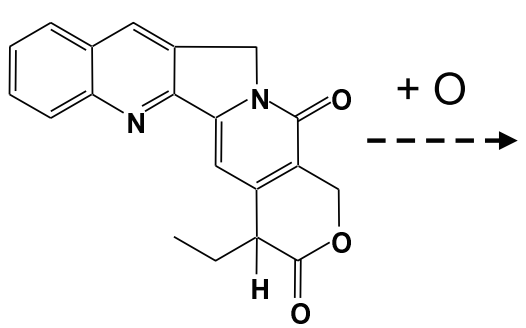

$\mathrm{C}_{20} \mathrm{H}_{16} \mathrm{~N}_{2} \mathrm{O}_{3}$

Ex MS: 332.1161

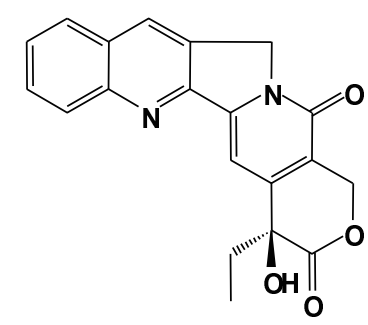

Camptothecin $\mathrm{C}_{20} \mathrm{H}_{16} \mathrm{~N}_{2} \mathrm{O}_{4}$ Ex MS: $\mathbf{3 4 8 . 1 1 1 0}$ 
Table 1. Peaks exhibiting positive correlation with $T D C$ expression

\begin{tabular}{|c|c|c|c|c|c|c|c|c|}
\hline \multirow[b]{2}{*}{$\begin{array}{l}\text { Peak } \\
\text { no. }\end{array}$} & \multirow[b]{2}{*}{$\begin{array}{l}\text { Correlation } \\
\text { coefficient } \\
(r)^{\mathrm{a}}\end{array}$} & \multirow[b]{2}{*}{$\begin{array}{l}\text { Retention } \\
\text { time }\end{array}$} & \multirow[b]{2}{*}{ Detected mass } & \multirow[b]{2}{*}{$\begin{array}{l}\text { Predicted } \\
\text { ion form }\end{array}$} & \multirow[b]{2}{*}{$\begin{array}{l}\text { Predicted } \\
\text { monoisotopic } \\
\text { mass }^{\mathrm{b}}\end{array}$} & \multicolumn{3}{|c|}{ KNApSAcK search } \\
\hline & & & & & & Formula & $\begin{array}{l}\text { Exact } \\
\text { average } \\
\text { mass }\end{array}$ & Annotated metabolite \\
\hline 82 & 0.74 & $32.49-32.63$ & $333.12341-333.12365$ & {$[\mathrm{M}+\mathrm{H}]^{+}$} & 332.1162 & & & \\
\hline 105 & 0.56 & $15.20-16.82$ & $359.19667-359.19684$ & {$[\mathrm{M}+\mathrm{H}]^{+}$} & 358.1895 & $\mathrm{C}_{20} \mathrm{H}_{26} \mathrm{~N}_{2} \mathrm{O}_{4}$ & 358.1893 & Gelsemicine \\
\hline $110-1$ & 0.76 & $5.77-5.87$ & $377.20722-377.20747$ & & & & & \\
\hline $110-2$ & 0.65 & $15.08-17.38$ & $377.20724-377.20745$ & & & & & \\
\hline $138-2$ & 0.40 & $33.61-33.72$ & $447.12873-447.12896$ & {$[\mathrm{M}+\mathrm{H}]^{+}$} & 446.1216 & $\mathrm{C}_{22} \mathrm{H}_{22} \mathrm{O}_{10}$ & 446.1213 & Wogonin 5-glucoside, Echioidin, and many others \\
\hline 148 & 0.75 & $31.01-31.18$ & $495.17646-495.17660$ & {$[\mathrm{M}+\mathrm{H}]^{+}$} & 494.1693 & & & \\
\hline 151 & 0.69 & $30.99-31.20$ & $496.17976-496.17998$ & & & & & \\
\hline $171-1$ & 0.50 & $18.28-18.50$ & $505.25489-505.25514$ & & & & & \\
\hline $171-2$ & 0.38 & $19.10-19.33$ & $505.25483-505.25509$ & & & & & \\
\hline $172-1$ & 0.49 & $18.29-18.49$ & $506.25816-506.25834$ & & & & & \\
\hline $172-2$ & 0.44 & $19.09-19.32$ & $506.25804-506.25828$ & & & & & \\
\hline 206 & 0.62 & $31.04-31.23$ & $549.18459-549.18500$ & {$[\mathrm{M}+\mathrm{Na}]^{+}$} & 526.1956 & $\mathrm{C}_{27} \mathrm{H}_{30} \mathrm{~N}_{2} \mathrm{O}_{9}$ & 526.1951 & Jorumycin, antibiotic UK 2B, Lyaloside \\
\hline
\end{tabular}

${ }^{a}$ Correlation coefficients were used to analyze the correlation between expression of the targeted gene and area of the selected peak.

${ }^{\mathrm{b}}$ Predicted ion form and predicted molecular mass matching the input $\mathrm{m} / \mathrm{z}$ with 5-ppm tolerance were generated using elements $\mathrm{C}, \mathrm{H}, \mathrm{N}$, and $\mathrm{O}$. 
Table 2. Peaks exhibiting negative correlation with $T D C$ expression

\begin{tabular}{|c|c|c|c|c|c|c|c|c|}
\hline \multirow[b]{2}{*}{$\begin{array}{l}\text { Peak } \\
\text { no. }\end{array}$} & \multirow[b]{2}{*}{$\begin{array}{l}\text { Correlation } \\
\text { coefficient } \\
(r)^{\mathrm{a}}\end{array}$} & \multirow[b]{2}{*}{$\begin{array}{l}\text { Retention } \\
\text { time }\end{array}$} & \multirow[b]{2}{*}{ Detected mass } & \multirow[b]{2}{*}{$\begin{array}{l}\text { Predicted } \\
\text { ion form }\end{array}$} & \multirow[b]{2}{*}{$\begin{array}{l}\text { Predicted } \\
\text { monoisotopic } \\
\text { mass }^{\mathrm{b}}\end{array}$} & \multicolumn{3}{|c|}{ KNApSAcK search } \\
\hline & & & & & & Formula & $\begin{array}{l}\text { Exact } \\
\text { average } \\
\text { mass }\end{array}$ & Annotated metabolite \\
\hline 65 & -0.71 & $29.81-29.90$ & $303.11133-303.11224$ & & & & & \\
\hline 128 & -0.70 & $31.86-32.10$ & $433.11307-433.11333$ & {$[\mathrm{M}+\mathrm{H}]^{+}$} & 432.1060 & $\mathrm{C}_{21} \mathrm{H}_{20} \mathrm{O}_{10}$ & 432.1057 & Vitexin, Kaempferol 7-rhamnoside, and many others \\
\hline 130 & -0.37 & $16.60-16.94$ & $437.16560-437.16583$ & {$[\mathrm{M}+\mathrm{H}]^{+}$} & 436.1584 & $\mathrm{C}_{18} \mathrm{H}_{28} \mathrm{O}_{12}$ & 436.1581 & Alpigenoside, Eleganoside $\mathrm{C}$, and many others \\
\hline $138-1$ & -0.71 & $32.00-32.20$ & $447.12848-447.12891$ & {$[\mathrm{M}+\mathrm{H}]^{+}$} & 446.1215 & $\mathrm{C}_{22} \mathrm{H}_{22} \mathrm{O}_{10}$ & 446.1213 & Wogonin 5-glucoside, Echioidin, and many others \\
\hline 140 & -0.63 & $24.92-25.23$ & $457.16825-457.16843$ & {$[\mathrm{M}+\mathrm{Na}]^{+}$} & 434.1791 & $\mathrm{C}_{32} \mathrm{H}_{22} \mathrm{~N}_{2}$ & 434.1783 & Biscryptolepine \\
\hline & & & & & & $\mathrm{C}_{19} \mathrm{H}_{30} \mathrm{O}_{11}$ & 434.17881 & Secologanin dimethyl acetal \\
\hline 141 & -0.55 & $20.09-20.52$ & $459.12677-459.12718$ & {$[\mathrm{M}+\mathrm{Na}]^{+}$} & 436.1377 & $\mathrm{C}_{21} \mathrm{H}_{24} \mathrm{O}_{10}$ & 436.1370 & Phloridzin, Catechin 3-O- $\alpha$-L-rhamnoside, and many others \\
\hline 180 & -0.44 & $22.19-22.44$ & $516.24933-516.24949$ & & & & & \\
\hline 220 & -0.59 & $32.02-32.21$ & $579.17112-579.17146$ & {$[\mathrm{M}+\mathrm{H}]^{+}$} & 578.1640 & $\mathrm{C}_{27} \mathrm{H}_{30} \mathrm{O}_{14}$ & 578.1636 & Kaempferitrin, Vitexin-2"-O-rhamnoside, and many others \\
\hline 222 & -0.60 & $32.02-32.19$ & $580.17445-580.17474$ & & & & & \\
\hline 229 & -0.60 & $31.97-32.24$ & $601.15300-601.15347$ & {$[\mathrm{M}+\mathrm{Na}]^{+}$} & 578.1641 & $\mathrm{C}_{27} \mathrm{H}_{30} \mathrm{O}_{14}$ & 578.1636 & Kaempferitrin, Vitexin-2"-O-rhamnoside, and many others \\
\hline 230 & -0.55 & $31.95-32.25$ & $602.15624-602.15676$ & & & & & \\
\hline
\end{tabular}

${ }^{a}$ Correlation coefficients were used to analyze the correlation between expression of the targeted gene and area of the selected peak.

${ }^{\mathrm{b}}$ Predicted ion form and predicted molecular mass matching the input $\mathrm{m} / \mathrm{z}$ with 5-ppm tolerance were generated using elements $\mathrm{C}$, $\mathrm{H}, \mathrm{N}$, and $\mathrm{O}$. 


\section{Graphical Abstract}

Suppression of camptothecin biosynthetic genes results in metabolic modification of secondary products in hairy roots of Ophiorrhiza pumila

Takashi Asano, Kanae Kobayashi, Emi Kashihara, Hiroshi Sudo, Ryosuke Sasaki, Yoko Iijima, Koh Aoki, Daisuke Shibata, Kazuki Saito*, and Mami Yamazaki ${ }^{*}$

Secondary metabolic flux was modified in the hairy roots of Ophiorrhiza pumila using RNAi technology to produce camptothecin, an anti-neoplastic monoterpenoid alkaloid, and related alkaloids.

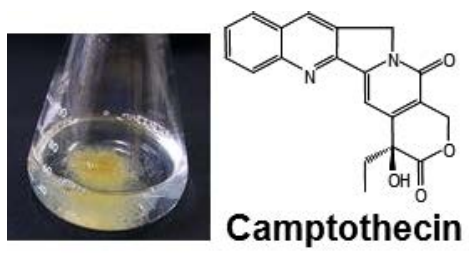

